\title{
ENTIRE SOLUTIONS OF QUASILINEAR SYMMETRIC SYSTEMS
}

\author{
Mostafa Fazly 1 \\ Department of Mathematical and Statistical Sciences, University of Alberta \\ Edmonton, Alberta, Canada T6G 2G1 \\ e-mail: fazly@ualberta.ca
}

Abstract. We study the following quasilinear elliptic system for all $i=1, \cdots, m$

$$
-\operatorname{div}\left(\Phi^{\prime}\left(\left|\nabla u_{i}\right|^{2}\right) \nabla u_{i}\right)=H_{i}(u) \text { in } \mathbb{R}^{n}
$$

where $u=\left(u_{i}\right)_{i=1}^{m}: \mathbb{R}^{n} \rightarrow \mathbb{R}^{m}$ and the nonlinearity $H_{i}(u) \in C^{1}\left(\mathbb{R}^{m}\right) \rightarrow \mathbb{R}$ is a general nonlinearity. Several celebrated operators such as the prescribed mean curvature, the Laplacian and the $p$-Laplacian operators fit in the above form, for appropriate $\Phi$. We establish a Hamiltonian identity of the following form for all $x_{n} \in \mathbb{R}$

$$
\int_{\mathbb{R}^{n-1}}\left(\sum_{i=1}^{m}\left[\frac{1}{2} \Phi\left(\left|\nabla u_{i}\right|^{2}\right)-\Phi^{\prime}\left(\left|\nabla u_{i}\right|^{2}\right)\left|\partial_{x_{n}} u_{i}\right|^{2}\right]-\tilde{H}(u)\right) d x^{\prime} \equiv C,
$$

where $x=\left(x^{\prime}, x_{n}\right) \in \mathbb{R}^{n}$ and $\tilde{H}$ is the antiderivative of $H=\left(H_{i}\right)_{i=1}^{m}$. This can be seen as a counterpart of celebrated pointwise inequalities provided by Caffarelli, Garofalo and Segala in [17] and by Modica in [2].

For the case of system of equations, that is when $m \geq 2$, we show that as long as

$$
\alpha \geq \alpha^{*}:=\inf _{s>0}\left\{\frac{2 s \Phi^{\prime}(s)}{\Phi(s)}\right\}
$$

the function $I_{\alpha}(r):=\frac{1}{r^{n-\alpha}} \int_{B_{r}} \sum_{i=1}^{m} \Phi\left(\left|\nabla u_{i}\right|^{2}\right)-2 \tilde{H}(u)$ is monotone nondecreasing in $r$. This in particular implies that for the prescribed mean curvature, the Laplacian, the $p$-Laplacian and operators the function $I_{\alpha}(r)$ is monotone when $\alpha \geq \alpha^{*}=2, \alpha \geq \alpha^{*}=2$ and $\alpha \geq \alpha^{*}=p$, respectively. We call this a weak monotonicity formula since for $m=1$ it is shown in 17] that $I_{\alpha}(r)$ is monotone when $\alpha \geq 1$, under certain conditions on $\Phi$.

We prove De Giorgi type results for $H$-monotone and stable solutions in two and three dimensions when the system is symmetric. The remarkable point is that gradients of all components of solutions are parallel and the angle between vectors $\nabla u_{i}$ and $\nabla u_{j}$ is precisely $\arccos \left(\frac{\left|\partial_{j} H_{i}\right|}{\partial_{j} H_{i}}\right)$. In addition, we provide an optimal Liouville theorem regarding radial stable solutions of the above system with a general nonlinearity when the system is symmetric. We announce several natural open problems in this context as well.

2010 Mathematics Subject Classification. 35J45, 35J93, 35J92, 35J50.

Keywords: Quasilinear elliptic systems, Hamiltonian identity, monotonicity formula, De Giorgi's conjecture, qualitative properties of solutions, prescribed mean curvature.

\section{Contents}

1. Introduction

2. Hamiltonian identities and monotonicity formulae

3. Geometric Poincaré and stability inequalities for systems

4. De Giorgi type results for symmetric systems

5. Liouville theorems for symmetric systems

References

\footnotetext{
${ }^{1}$ The author is partially supported by National Sciences and Engineering Research Council of Canada (NSERC) Discovery Grant\#RES0020463.
} 


\section{INTRODUCTION}

In [17], Caffarelli, Garofalo and Segala studied the following class of quasilinear equations arising in geometry

$$
\operatorname{div}\left(\Phi^{\prime}\left(|\nabla u|^{2}\right) \nabla u\right)=f(u) \quad \text { in } \mathbb{R}^{n},
$$

where $f$ is a $C^{1}(\mathbb{R})$ and $\Phi \in C^{2}\left(\mathbb{R}^{+}\right)$satisfies certain conditions that follow. Note that for the case of $\Phi(s)=s$ the above equation is the standard semilinear elliptic equation. The prescribed mean curvature equation and the $p$-Laplacian equation, i.e.

$$
\begin{aligned}
& \operatorname{div}\left(\frac{\nabla u}{\sqrt{1+|\nabla u|^{2}}}\right)=f(u) \text { in } \mathbb{R}^{n}, \\
& \operatorname{div}\left(\left(\epsilon+|\nabla u|^{2}\right)^{\frac{p-2}{2}} \nabla u\right)=f(u) \text { in } \mathbb{R}^{n} \text { for } \epsilon>0,
\end{aligned}
$$

respectively, fit in the form of (1.1) where $\Phi$ is given respectively by

$$
\begin{aligned}
& \Phi(s)=2(\sqrt{1+s}-1), \\
& \Phi(s)=\frac{2}{p}\left((\epsilon+s)^{\frac{p}{2}}-\epsilon^{\frac{p}{2}}\right) .
\end{aligned}
$$

Throughout this paper we shall assume that $\Phi(s), \Phi^{\prime}(s)$ and $\Phi^{\prime}(s)+2 \Phi^{\prime \prime}(s) s$ are positive when $s>0$. In addition, without loss of generality let $\Phi(0)=0$. Borrowing notations from [17], we shall refer to the following conditions often in this paper. Note that $\Phi$ in (1.4) and (1.5) satisfies these conditions, respectively, Condition (A). There exist positive constants $C_{1}, C_{2}$ and $\epsilon \geq 0$ such that $\Phi \in C^{2}\left(\mathbb{R}^{+}\right)$and for every $\eta, \zeta \in \mathbb{R}^{n}$

$$
\begin{aligned}
& C_{1}(\epsilon+|\eta|)^{-1} \leq \Phi^{\prime}\left(|\eta|^{2}\right) \leq C_{2}(\epsilon+|\eta|)^{-1}, \\
& C_{1}(\epsilon+|\eta|)^{-1}\left|\zeta^{\prime}\right|^{2} \leq \sum_{i, j=1}^{m} a_{i, j}(\eta) \zeta_{i} \zeta_{j} \leq C_{2}(\epsilon+|\eta|)^{-1}\left|\zeta^{\prime}\right|^{2},
\end{aligned}
$$

where $\zeta^{\prime}=\left(\zeta, \zeta_{n+1}\right) \in \mathbb{R}^{n+1}$ is orthogonal to the vector $(-\eta, 1) \in \mathbb{R}^{n+1}$.

Condition (B). There exist $p>1, \epsilon \geq 0$ and positive constants $C_{1}, C_{2}$ such that $\Phi \in C^{2}\left(\mathbb{R}^{+}\right)$that for every $\eta, \zeta \in \mathbb{R}^{n}$,

$$
\begin{aligned}
& C_{1}(\epsilon+|\eta|)^{p-2} \leq \Phi^{\prime}\left(|\eta|^{2}\right) \leq C_{2}(\epsilon+|\eta|)^{p-2}, \\
& C_{1}(\epsilon+|\eta|)^{p-2}|\zeta|^{2} \leq \sum_{i, j=1}^{m} a_{i, j}(\eta) \zeta_{i} \zeta_{j} \leq C_{2}(\epsilon+|\eta|)^{p-2}|\zeta|^{2},
\end{aligned}
$$

where $a_{i, j}$ in (1.9) and (1.7) are given by

$$
a_{i, j}(\eta):=2 \Phi^{\prime \prime}\left(|\eta|^{2}\right) \eta_{i} \eta_{j}+\Phi^{\prime}\left(|\eta|^{2}\right) \delta_{i j} .
$$

One of the main results provided in [17] is the following pointwise inequality. Note that this is a counterpart of the pointwise estimate given by Modica in [42] for the case of $\Phi(s)=s$.

Theorem A. Suppose that $f \in C^{2}(\mathbb{R})$ with $F \geq 0$ and suppose that one of the following conditions hold

(i) Condition (A) holds and $u \in W_{\text {loc }}^{1, p}\left(\mathbb{R}^{n}\right) \cap L^{\infty}\left(\mathbb{R}^{n}\right)$ is a solution to (1.1)

(ii) Condition (B) holds and $u \in C^{2}\left(\mathbb{R}^{n}\right) \cap L^{\infty}\left(\mathbb{R}^{n}\right)$ is a solution to (1.1) and in additon $|\nabla u| \in L^{\infty}\left(\mathbb{R}^{n}\right)$.

Then for every $x$

$$
2 \Phi^{\prime}\left(|\nabla u|^{2}\right)|\nabla u|^{2}-\Phi\left(|\nabla u|^{2}\right) \leq 2 F(u) .
$$

In particular, the following pointwise estimates hold for specific $\Phi$.

- Suppose that $\Phi(s)=s$, then

$$
|\nabla u|^{2} \leq 2 F(u) \text { in } \mathbb{R}^{n},
$$

where $u$ is a bounded solution of the semilinear equation $\Delta u=f(u)$ in $\mathbb{R}^{n}$, provided by Modica in [42]. 
- Let $\Phi(s)=2(\sqrt{1+s}-1)$. Then

$$
\frac{\sqrt{1+|\nabla u|^{2}}-1}{\sqrt{1+|\nabla u|^{2}}} \leq F(u) \text { in } \mathbb{R}^{n}
$$

for bounded solutions of the prescribed mean curvature equation that is $\operatorname{div}\left(\frac{\nabla u}{\sqrt{1+|\nabla u|^{2}}}\right)=f(u)$ in $\mathbb{R}^{n}$.

- Suppose that $\Phi(s)=\frac{2}{p} s^{\frac{p}{2}}$. Then

$$
|\nabla u|^{p} \leq \frac{p}{p-1} F(u) \text { in } \mathbb{R}^{n},
$$

where $u$ is a bounded solution of the $p$-Laplace equation $\operatorname{div}\left(|\nabla u|^{p-2} \nabla u\right)=f(u)$ in $\mathbb{R}^{n}$.

We study classical solutions of the following quasilinear system of equations

$$
-\operatorname{div}\left(\Phi^{\prime}\left(\left|\nabla u_{i}\right|^{2}\right) \nabla u_{i}\right)=H_{i}(u) \text { in } \mathbb{R}^{n},
$$

where $u=\left(u_{i}\right)_{i=1}^{m}: \mathbb{R}^{n} \rightarrow \mathbb{R}^{m}$ and $H_{i}(u) \in C^{1}\left(\mathbb{R}^{m}\right) \rightarrow \mathbb{R}$ for all $i=1, \cdots, m$. The above system has variational structure and the associated energy functional is given by

$$
E(u)=\int \sum_{i=1}^{m} \frac{1}{2} \Phi\left(\left|\nabla u_{i}\right|^{2}\right)-\tilde{H}(u),
$$

where $\tilde{H}$ is defined such that $\partial_{i} \tilde{H}(u)=H_{i}(u)$. Throughout this paper we use the notation $u=\left(u_{i}\right)_{i=1}^{m}$, $H(u)=\left(H_{i}(u)\right)_{i=1}^{m}$ and $\partial_{j} H_{i}(u)=\frac{\partial H_{i}(u)}{\partial u_{j}}$. We assume that $\partial_{i} H_{j}(u) \partial_{j} H_{i}(u)>0$ for $1 \leq i \leq j \leq m$. The next definition is the notion of the symmetric systems, introduced by the author in [34]. Symmetric systems play a fundamental role throughout this paper when we deal with the energy functional given in (1.16) and when we study system (1.15) with a general nonlinearity $H(u)$. Note that for the scalar equation case, that is when $m=1$, (1.15) is clearly symmetric.

Definition 1.1. We call system (1.15) symmetric if the matrix of partial derivatives of all components of $H$ given by

$$
\mathbb{H}:=\left(\partial_{i} H_{j}(u)\right)_{i, j=1}^{m},
$$

is symmetric.

Hamiltonian identities are quite well-known in both mathematics and physics as important tools to study qualitative behaviour of entire solutions of differential equations and systems. They often directly or indirectly lead to certain properties which could be of great importance in the fields as well, such as monotonicity formulae. Consider the following symmetric system of ordinary differential equations that is a particular case of (1.15),

$$
-u_{i}^{\prime \prime}=\partial_{i} H(u) \text { in } \mathbb{R} .
$$

It is straightforward to see that the following Hamiltonian identity holds for solutions of (1.18)

$$
\frac{1}{2} \sum_{i=1}^{m} u_{i}^{\prime 2}+H(u) \equiv C \text { in } \mathbb{R}
$$

where $C$ is a constant. Equivalently, one can rewrite (1.18) in the form of a first order Hamiltonian system

$$
\left\{\begin{aligned}
-u_{i}^{\prime} & =\partial_{v_{i}} \bar{H}(u, v) \text { in } \mathbb{R}, \\
-v_{i}^{\prime} & =-\partial_{u_{i}} \bar{H}(u, v) \text { in } \mathbb{R},
\end{aligned}\right.
$$

where $\bar{H}(u, v)=\frac{1}{2} \sum_{i=1}^{m} v_{i}^{2}+H(u)$. Note that $\bar{H}(u, v) \equiv C$ on trajectories of solutions. These equations generalize Newton's third law that is $F=m a$ to system of equations where the momentum is not simply mass times velocity. The Hamiltonian $\bar{H}(u, v)$ normally represents the total energy of the system. We refer interested readers to [7, 40] for some original information regarding physical meaning of the system and to 29, 41] and references therein for variational theory of Hamiltonian systems. 
Gui in [39] considered the gradient system $-\Delta u_{i}=\partial_{i} H(u)$, that is a higher-dimensional counterpart of (1.18), and established the following Hamiltonian identity,

$$
\int_{R^{n-1}}\left[\frac{1}{2} \sum_{i=1}^{m}\left(\left|\nabla_{x^{\prime}} u_{i}\right|^{2}-\left|\partial_{x_{n}} u_{i}\right|^{2}\right)-H(u(x))\right] d x^{\prime} \equiv C,
$$

for $x=\left(x^{\prime}, x_{n}\right) \in \mathbb{R}^{n-1} \times \mathbb{R}$. In this paper, we provide an extension of this inequality for solutions of quasilinear symmetric system (1.15). One might expect, at the first glance, that just replacing derivative terms with $\Phi\left(\left|\nabla_{x^{\prime}} u_{i}\right|^{2}\right)-\Phi\left(\left|\partial_{x_{n}} u_{i}\right|^{2}\right)$ could simply give the Hamiltonian identity for solutions of (1.15). Instead, the identity follows the structure of the pointwise estimate provided by Caffarelli, Garofalo and Segala in [17] and it is of the form

$$
\int_{\mathbb{R}^{n-1}}\left(\sum_{i=1}^{m}\left[\frac{1}{2} \Phi\left(\left|\nabla u_{i}\right|^{2}\right)-\Phi^{\prime}\left(\left|\nabla u_{i}\right|^{2}\right)\left|\partial_{x_{n}} u_{i}\right|^{2}\right]-\tilde{H}(u(x))\right) d x^{\prime} \equiv C .
$$

Note that when $\Phi(s)=s$, the identity (1.21) recovers (1.20). This then explains why the difference of partial derivatives that is $\left|\nabla_{x^{\prime}} u_{i}\right|^{2}-\left|\partial_{x_{n}} u_{i}\right|^{2}$ appears in (1.20).

If we set $\Phi$ to be the ones given in (1.4) and (1.5) we can have the Hamiltonian identity for the prescribed mean curvature equation and the $p$-Laplacian equation, respectively. Let us mention this remarkable point again that the Hamiltonian identity (1.21) has a very similar structure as pointwise estimates (1.11) and (1.12), provided by Caffarelli, Garofalo and Segala in [17] and by Modica in [42]. Therefore, (1.21) can be seen as a counterpart of (1.11) for the case of system of equations, i.e. $m \geq 2$.

The Hamiltonian identity (1.21) motivates us to look for a monotonicity formula for solutions of (1.15). So, set

$$
I_{\alpha}(r):=\frac{1}{r^{n-\alpha}} \int_{B_{r}}\left[\sum_{i=1}^{m} \Phi\left(\left|\nabla u_{i}\right|^{2}\right)-2 \tilde{H}(u)\right] .
$$

For the case of scalar equations, that is when $m=1$, it is proved by Caffarelli, Garofalo and Segala in [17] that when $\Phi$ satisfies one of conditions (A) or (B) then the function $I_{\alpha}(r)$ is monotone nondecreasing in $r$ when $\alpha \geq 1$. They have used the pointwise inequality (1.11) to establish this monotonicity formula. For the case of $m \geq 2$, we show that $I_{\alpha}(r)$ is monotone nondecreasing in $r$ when

$$
\alpha \geq \alpha^{*}:=\inf _{s>0}\left\{\frac{2 s \Phi^{\prime}(s)}{\Phi(s)}\right\}
$$

We call this a weak monotonicity formula since the constant $\alpha^{*}$ must be greater than one, due to some general assumptions on $\Phi$. To clarify this, define an auxiliary function $h(s):=-2 \Phi^{\prime}(s) s+\alpha \Phi(s)$ in the light of (1.23). Note that from assumptions on $\Phi$, i.e. $2 s \Phi^{\prime \prime}(s)+\Phi^{\prime}(s)>0$ when $s>0$ and $h(0)=\alpha \Phi(0)=0$ one can see that $h^{\prime}(s)=-\left[2 s \Phi^{\prime \prime}(s)+\Phi^{\prime}(s)\right]+(\alpha-1) \Phi^{\prime}(s)$ is negative when $\alpha \leq 1$. For certain functions $\Phi$, one can get the Laplacian, the $p$-Laplacian and the prescribed mean curvature operators and then the function $I_{\alpha}(r)$ is monotone in $r$ when $\alpha \geq \alpha^{*}=2, \alpha \geq \alpha^{*}=p$ and $\alpha \geq \alpha^{*}=2$, respectively, see Corollary 2.2. On the other hand, for both cases of scalar equations and system of equations, i.e. $m \geq 1$, it is shown in Theorem 2.4 that the following upper bound on the energy holds,

$$
\int_{B_{R}}\left[\sum_{i=1}^{m} \Phi\left(\left|\nabla u_{i}\right|^{2}\right)-2 \tilde{H}(u)+2 \tilde{H}(a)\right] d x \leq C R^{n-1},
$$

where $\lim _{x_{n} \rightarrow \infty} u_{i}\left(x^{\prime}, x_{n}\right)=a_{i}$ for all $x=\left(x^{\prime}, x_{n}\right) \in \mathbb{R}^{n}$ and $a=\left(a_{i}\right)_{i=1}^{m}$. This implies that for the case of system of equations, $m \geq 2$, the strong monotonicity formula, that is when $\alpha \geq 1$, should hold just like in the case of scalar equations for $m=1$. This remains as an open problem. Note also that conditions (A) and (B) are not necessary for our monotonicity formula when $m \geq 2$.

We apply monotonicity formulae to establish Liouville theorems for solutions of (1.15) with a finite energy. We refer interested readers to Alikakos in [3, 4] and to Alikakos and Fusco in [5], to Caffarelli, Garofalo and Segala in [17] and to Farina in [30, 32] regarding Liouville theorems for various equations and systems with a finite energy. Note that the above monotonicity formulae, in both weak and strong forms, are related to the ones given for harmonic maps by Schoen and Uhlenbeck in [50] and for minimal surfaces by Simon in 
[51, by Ecker in [28] and by Schoen in [49] and for elliptic equations by Caffarelli and Lin in [18], by Modica in [43] and references therein.

Regarding the scalar equation case, in this context, monotonicity of a solution $u$ is straightforward to define and it refers to solutions that are monotone in one direction, e.g. when $\partial_{x_{n}} u_{i}$ does not change sign, see [2, 6, 9, 17, 23, 24, 30 33, 37, 38, 44, 47] and references therein. However, the notion of monotonicity of solutions for the case of system of equations, that is when $m \geq 2$, seems to be slightly more sophisticated. Ghoussoub and the author in [35] introduced the following concept of monotonicity for the case of system of equations. Note that the sign of partial derivatives of the nonlinearity $H$ could potentially have an impact on the monotonicity of solutions. This motivates us to call this notion as $H$-monotonicity.

Definition 1.2. A solution $u=\left(u_{k}\right)_{k=1}^{m}$ of (1.15) is said to be $H$-monotone if the following holds,

(i) For every $1 \leq i \leq m$, each $u_{i}$ is strictly monotone in the $x_{n}$-variable (i.e., $\partial_{x_{n}} u_{i} \neq 0$ ).

(ii) For all $i \leq j$, we have

$$
\partial_{j} H_{i}(u) \partial_{x_{n}} u_{i}(x) \partial_{x_{n}} u_{j}(x)>0 \text { for all } x \in \mathbb{R}^{n} .
$$

See [34, 35] for more details.

Note also that in the assumption (ii) of the $H$-monotonicity each of $\partial_{j} H_{i}(u), \partial_{x_{n}} u_{i}(x)$ and $\partial_{x_{n}} u_{j}(x)$ has a fixed sign and the multiplication must be positive. This implies a combinatorial assumption on the system (1.15). We refer to systems that admit such an assumption as orientable systems. For an example, consider $m=2$ then for cross type solutions, i.e. $\partial_{x_{n}} u_{1}>0$ and $\partial_{x_{n}} u_{2}<0$, we are required to set $\partial_{1} H_{2}(u), \partial_{2} H_{1}(u)<0$. If we set $H_{1}(u)=H_{2}(u)=-\frac{1}{2} u_{1}^{2} u_{2}^{2}$ then this gives a two component system of equations that arrises in Bose-Einstein condensates, see [1] and references therein.

The next definition is the notion of stable solutions for the case of system of equations.

Definition 1.3. A solution $u=\left(u_{k}\right)_{k=1}^{m}$ of (1.15) is called stable when there exists a sequence of functions $\phi=\left(\phi_{k}\right)_{k=1}^{m}$ such that each $\phi_{i}$ does not change sign and $\partial_{j} H_{i}(u) \phi_{j} \phi_{i}>0$ for all $i, j=1, \cdots, m$. In addition, $\phi$ satisfies the following

$$
-\operatorname{div}\left(\mathcal{A}\left(\nabla u_{i}\right) \nabla \phi_{i}\right)=\sum_{j=1}^{m} \partial_{j} H_{i}(u) \phi_{j} \quad \text { in } \mathbb{R}^{n},
$$

where for any $\eta \in \mathbb{R}^{n}$ the matrix $\mathcal{A}(\eta)$ is defined by $\mathcal{A}(\eta):=\left(a_{i, j}(\eta)\right)_{i, j=1}^{n}$ for $a_{i, j}(\eta)$ in (1.10).

Let us mention that the notion of stability can be given for weak solutions as

$$
\int \mathcal{A}\left(\nabla u_{i}\right) \nabla \phi_{i} \cdot \nabla \zeta_{i}=\sum_{j=1}^{m} \partial_{j} H_{i}(u) \phi_{j} \zeta_{i},
$$

where $\zeta=\left(\zeta_{i}\right)_{i=1}^{m}$ is a sequence of test functions. Accordingly one can see that all results provided in the present paper are valid for weak solution as well. For the sake of simplicity in notation, we present our results for classical solutions. We refer to 21] and references therein for the use of stability for nonlinear elliptic eigenvalue problems.

Since (1.26) is a linearization of (1.15), one can see that every $H$-monotone solution is a stable solution via differentiating (1.15) with respect to $x_{n}$. The notion of stability as well as the monotonicity formula for $I_{\alpha}(r)$ when $\alpha \geq 1$ and the pointwise inequality (1.12), provided by Modica, play key role in settling the De Giorgi's conjecture (1978), see [23]. The conjecture states that bounded monotone solutions of Allen-Cahn equation are one-dimensional solutions at least up to eight dimensions. There is an affirmative answer to this conjecture for almost all dimensions. More precisely, for two dimensions Ghoussoub and Gui in [37] and for three dimensions Ambrosio and Cabré in [6] and with Alberti in [2] gave a proof to this conjecture not only for Allen-Cahn equation but also for any equation of the form $-\Delta u=f(u)$ where $f$ is a general nonlinearity that is locally Lipschitz. For dimensions $4 \leq n \leq 8$ there are various partial results under certain extra (natural) assumptions on solutions by Ghoussoub and Gui in [38], by Savin in [47] and references therein. Note that there is an example by del Pino, Kowalczyk and Wei in 24] showing that eight dimensions is the critical dimension. In two dimensions regarding the De Giorgi's conjecture, we refer to Farina, Sciunzi and Valdinoci in [33] for a geometrical approach and to Modica and Mortola in [44] for some partial results under 
the additional assumption that the level sets of solutions are the graphs of an equi-Lipschitzian family of functions.

In [17, 22, 33], authors considered quasilinear scalar equations of the form of (1.15) when $m=1$ and provided one-dimensional symmetry and De Giorgi type results. Note that Ghoussoub and the author in [35] provided De Giorgi type results for elliptic systems of the form $-\Delta u_{i}=\partial_{i} H(u)$ in lower dimensions for a general nonlinearity $H$.

In this paper, we first provide a geometric Poincaré inequality and a linear Liouville theorem for stable and $H$-monotone solutions of the quasilinear system (1.15). Then we apply these to conclude De Giorgi type results for $H$-monotone and stable solutions in two and three dimensions when the system is symmetric. For coupled systems, that is when not all $\partial_{j} H_{i}$ vanish for $1 \leq i<j \leq m$, it is natural to expect that there should be a relation between two arbitrary components $u_{i}$ and $u_{j}$. In this regard, we show that gradients of all components of solutions are parallel and the angle in between $\nabla u_{i}$ and $\nabla u_{j}$ is precisely arccos $\left(\frac{\left|\partial_{j} H_{i}\right|}{\partial_{j} H_{i}}\right)$ when $\partial_{j} H_{i} \neq 0$. This is a consequence of the geometric Poincaré inequality, see Theorem 3.1 .

The main focus of the present paper is the study of qualitative properties of solutions of system (1.15) with a general nonlinearity. In this paper, we prove a Liouville theorem for bounded stable solutions of (1.15) in dimensions $n \leq 4$ for a general nonlinearity $H=\left(H_{i}\right)_{i=1}^{m}$ whenever each $H_{i}$ is nonnegative. To do so, we suppose that $\Phi$ satisfies either condition (A) or (B). Note that for the case of semilinear equations similar results are given by Dupaigne and Farina in [27] and for the case of semilinear systems by Ghoussoub and the author in [35]. In addition, we give a classification of radial stable solutions of symmetric system (1.15) when $\Phi(s)=\frac{2}{p} s^{\frac{p}{2}}$ for all $m \geq 1$. More precisely, we show that there exists a positive constant $C_{n, m, p}$ such that for any $r$, the following pointwise lower bound holds,

$$
\sum_{i=1}^{m}\left|u_{i}(r)\right| \geq C_{n, m, p} \begin{cases}r^{\frac{1}{p}\left(p+2-n+2 \sqrt{\frac{n-1}{p-1}}\right),} & \text { if } n \neq \frac{4 p}{p-1}+p \\ \log r, & \text { if } n=\frac{4 p}{p-1}+p .\end{cases}
$$

This in particular implies that bounded radial stable solutions must be constant in dimensions $1 \leq n<$ $\frac{4 p}{p-1}+p$. The notion of symmetric systems seems to be essential to study (1.15) with a general nonlinearity. Note also that the critical dimension $n=\frac{4 p}{p-1}+p$ for radial solutions is much higher than the dimension $n=4$ derived for not necessarily radial solutions. Let us mention that for the case of semilinear equations, that is $\Phi(s)=s$ and $m=1$, it is proved by Cabré-Capella [14, 15] and Villegas [52] that any bounded radial stable solution of (1.15) has to be constant provided $1 \leq n<10$ when $H \in C^{1}(\mathbb{R})$ is a general nonlinearity. In addition, for the case of scalar equation and when $\Phi(s)=\frac{2}{p} s^{\frac{p}{2}}$, a counterpart of the above Liouvillle theorem is provided in [16, 19].

Here is how this paper is organized. Shortly after, in Section 2 we provide a Hamiltonian identity for solutions of system (1.15). We also prove monotonicity formulae and we apply it to establish a Liouville theorem for solutions with finite energy. A few open problems are provided in this section as well. Section 3 is devoted to some estimates needed to prove De Giorgi type results and Liouville theorems in next sections. We start the section with a stability inequality and then we apply this inequality to establish a geometric Poincaré inequality. In Section 4, we establish De Giorgi type results for $H$-monotone and stable solutions of symmetric system (1.15). In addition, we apply the geometric Poincaré inequality, provided in Section 4. to find a relation between gradients of all components of solutions of (1.15). Finally in Section 5, we prove Liouville theorems for stable solutions of (1.15) with a general nonlinearity, with on case requiring the solutions to be also radial. The concept of symmetric systems seems to be crucial to prove such an optimal Liouville theorem for radial solutions.

\section{Hamiltonian identities AND MONOTONICITY FORMULAE}

We start this section by the following Hamiltonian identity.

Theorem 2.1. Suppose that $u=\left(u_{i}\right)_{i=1}^{m}$ is a solution of (1.15) and let $x=\left(x^{\prime}, x_{n}\right) \in \mathbb{R}^{n-1} \times \mathbb{R}$. Then there exists a constant $C$ such that the following Hamiltonian identity holds for every $x_{n} \in \mathbb{R}$

$$
\int_{\mathbb{R}^{n-1}}\left(\sum_{i=1}^{m}\left[\frac{1}{2} \Phi\left(\left|\nabla u_{i}\right|^{2}\right)-\Phi^{\prime}\left(\left|\nabla u_{i}\right|^{2}\right)\left|\partial_{x_{n}} u_{i}\right|^{2}\right]-\tilde{H}(u)\right) d x^{\prime} \equiv C,
$$


when the above integral is finite for at least one value of $x_{n}$ and in addition the integral in (2.10) below tends to zero as $R$ goes to infinity along a sequence.

Proof. Suppose that $x=\left(x^{\prime}, x_{n}\right) \in \mathbb{R}^{n}$ and assume that $B_{R}(0)$ is a ball of radius $R$ in $\mathbb{R}^{n-1}$. Define $\Gamma: \mathbb{R} \rightarrow \mathbb{R}$ as

$$
\Gamma_{R}\left(x_{n}\right):=\int_{B_{R}(0)}\left(\sum_{i=1}^{m}\left[\frac{1}{2} \Phi\left(\left|\nabla u_{i}\right|^{2}\right)-\Phi^{\prime}\left(\left|\nabla u_{i}\right|^{2}\right)\left|\partial_{x_{n}} u_{i}\right|^{2}\right]-\tilde{H}(u)\right) d x^{\prime} .
$$

Differentiating $\Gamma$ with respect to $x_{n}$ we get

$$
\begin{aligned}
\Gamma_{R}^{\prime}\left(x_{n}\right)= & \sum_{i=1}^{m} \int_{B_{R}(0)}\left\{\frac{1}{2} \partial_{x_{n}}\left[\Phi\left(\left|\nabla u_{i}\right|^{2}\right)\right]-\partial_{x_{n}}\left[\Phi^{\prime}\left(\left|\nabla u_{i}\right|^{2}\right)\left|\partial_{x_{n}} u_{i}\right|^{2}\right]\right. \\
& \left.-H_{i}(u) \partial_{x_{n}} u_{i}\right\} d x^{\prime} \\
=: & \sum_{i=1}^{m} \int_{B_{R}(0)}\left\{\Gamma^{1}(x)+\Gamma^{2}(x)+\Gamma^{3}(x)\right\} d x^{\prime} .
\end{aligned}
$$

In what follows we simplify the above three terms, appeared in the right-hand side of (2.3). Note that

$$
\partial_{x_{n}}\left[\Phi\left(\left|\nabla u_{i}\right|^{2}\right)\right]=2 \Phi^{\prime}\left(\left|\nabla u_{i}\right|^{2}\right) \nabla u_{i} \cdot \nabla \partial_{x_{n}} u_{i} .
$$

Therefore,

$$
\Gamma^{1}(x)=\Phi^{\prime}\left(\left|\nabla u_{i}\right|^{2}\right) \nabla u_{i} \cdot \nabla \partial_{x_{n}} u_{i} .
$$

Similarly,

$$
\begin{aligned}
\Gamma^{2}(x)= & -2 \Phi^{\prime \prime}\left(\left|\nabla u_{i}\right|^{2}\right) \nabla u_{i} \cdot \nabla \partial_{x_{n}} u_{i}\left|\partial_{x_{n}} u_{i}\right|^{2} \\
& -2 \Phi^{\prime}\left(\left|\nabla u_{i}\right|^{2}\right) \partial_{x_{n}} u_{i} \partial_{x_{n} x_{n}}^{2} u_{i} .
\end{aligned}
$$

We now apply (1.15) to simplify $\Gamma^{3}$,

$$
\begin{aligned}
\Gamma^{3}(x)=\operatorname{div}\left(\Phi^{\prime}\left(\left|\nabla u_{i}\right|^{2}\right) \nabla u_{i}\right) \partial_{x_{n}} u_{i}= & \operatorname{div}_{x^{\prime}}\left(\Phi^{\prime}\left(\left|\nabla u_{i}\right|^{2}\right) \nabla_{x^{\prime}} u_{i}\right) \partial_{x_{n}} u_{i} \\
& +2 \Phi^{\prime \prime}\left(\left|\nabla u_{i}\right|^{2}\right) \nabla u_{i} \cdot \nabla \partial_{x_{n}} u_{i}\left|\partial_{x_{n}} u_{i}\right|^{2} \\
& +\Phi^{\prime}\left(\left|\nabla u_{i}\right|^{2}\right) \partial_{x_{n}} u_{i} \partial_{x_{n} x_{n}}^{2} u_{i} .
\end{aligned}
$$

Adding (2.5), (2.6) and (2.7) we get

$$
\begin{aligned}
\Gamma^{1}(x)+\Gamma^{2}(x)+\Gamma^{3}(x)= & \operatorname{div}_{x^{\prime}}\left(\Phi^{\prime}\left(\left|\nabla u_{i}\right|^{2}\right) \nabla_{x^{\prime}} u_{i}\right) \partial_{x_{n}} u_{i} \\
& +\Phi^{\prime}\left(\left|\nabla u_{i}\right|^{2}\right) \partial_{x_{n}} u_{i} \partial_{x_{n} x_{n}}^{2} u_{i} .
\end{aligned}
$$

Substituting (2.8) in (2.3) and applying the divergence theorem we obtain

$$
\Gamma_{R}^{\prime}\left(x_{n}\right)=\sum_{i=1}^{m} \int_{\partial B_{R}(0)} \Phi^{\prime}\left(\left|\nabla u_{i}\right|^{2}\right) \partial_{\nu_{x^{\prime}}} u_{i} \partial_{x_{n}} u_{i} .
$$

Suppose that the integral in (2.1) is finite when $x_{n}=0$. Then,

$$
\Gamma_{R}\left(x_{n}\right)-\Gamma_{R}(0)=\sum_{i=1}^{m} \int_{0}^{x_{n}} \int_{\partial B_{R}(0)} \Phi^{\prime}\left(\left|\nabla u_{i}\right|^{2}\right) \partial_{\nu_{x^{\prime}}} u_{i} \partial_{x_{n}} u_{i}
$$

Taking the limit of the above when $R \rightarrow \infty$ finishes the proof.

When $\Phi$ is the identity function, the system of equations (1.15) is a semilinear system of the following form

$$
-\Delta u_{i}=H_{i}(u) \text { in } \mathbb{R}^{n} .
$$

Therefore, Theorem 2.1 implies that the following Hamiltonian identity holds,

$$
\left.\int_{\mathbb{R}^{n-1}}\left(\sum_{i=1}^{m} \frac{1}{2}\left[\left|\nabla_{x^{\prime}} u_{i}\right|^{2}\right)-\left|\partial_{x_{n}} u_{i}\right|^{2}\right]-\tilde{H}(u)\right) d x^{\prime} \equiv C .
$$


Note that (2.11) is given by Gui in [39]. Here we have Hamiltonian identities for the mean curvature system as well as the $p$-Laplacian system.

Corollary 2.1. Suppose that assumptions of Theorem 2.1 hold.

(i) Let $\Phi(s)=2(\sqrt{s+1}-1)$. Then (1.15) reads

$$
-\operatorname{div}\left(\frac{\nabla u_{i}}{\sqrt{1+\left|\nabla u_{i}\right|^{2}}}\right)=H_{i}(u) \quad \text { in } \mathbb{R}^{n} .
$$

For any $x_{n} \in \mathbb{R}$, this Hamiltonian identity holds,

$$
\int_{\mathbb{R}^{n-1}}\left(\sum_{i=1}^{m}\left[\frac{1+\left|\nabla_{x^{\prime}} u_{i}\right|^{2}-\sqrt{1+\left|\nabla u_{i}\right|^{2}}}{\sqrt{1+\left|\nabla u_{i}\right|^{2}}}\right]-\tilde{H}(u)\right) d x^{\prime} \equiv C .
$$

(ii) Let $\Phi(s)=\frac{2}{p} s^{\frac{p}{2}}$. Then (1.15) reads

$$
-\operatorname{div}\left(\left|\nabla u_{i}\right|^{p-2} \nabla u_{i}\right)=H_{i}(u) \quad \text { in } \mathbb{R}^{n}
$$

For any $x_{n} \in \mathbb{R}$, this Hamiltonian identity holds,

$$
\int_{\mathbb{R}^{n-1}}\left(\sum_{i=1}^{m}\left|\nabla u_{i}\right|^{p-2}\left[\frac{1}{p}\left|\nabla_{x^{\prime}} u_{i}\right|^{2}-\frac{p-1}{p}\left|\partial_{x_{n}} u_{i}\right|^{2}\right]-\tilde{H}(u)\right) d x^{\prime} \equiv C .
$$

where $C$ is a constant.

Note that for a specific nonlinearity of the form $H_{i}(u)=u_{i}\left(1-\sum_{i=1}^{m} u_{i}^{2}\right)$, the original system of equations $(1.15)$ is

$$
-\operatorname{div}\left(\Phi^{\prime}\left(\left|\nabla u_{i}\right|^{2}\right) \nabla u_{i}\right)=u_{i}\left(1-\sum_{i=1}^{m} u_{i}^{2}\right) \quad \text { in } \mathbb{R}^{n}
$$

This is a quasilinear Ginzburg-Landau system where $u=\left(u_{i}\right)_{i=1}^{m}$ for $u_{i}: \mathbb{R}^{n} \rightarrow \mathbb{R}$. For the semilinear case and $m=2$, see [12, 32]. Since $\tilde{H}=-\frac{1}{4}\left(1-\sum_{i=1}^{m} u_{i}^{2}\right)^{2}$ is an antiderivative of $H$, the following Hamiltonian identity holds, as long as conditions of Theorem 2.1 are satisfied, for any $x_{n} \in \mathbb{R}$

$$
\int_{\mathbb{R}^{n-1}}\left\{\sum_{i=1}^{m}\left[\frac{1}{2} \Phi\left(\left|\nabla u_{i}\right|^{2}\right)-\Phi^{\prime}\left(\left|\nabla u_{i}\right|^{2}\right)\left|\partial_{x_{n}} u_{i}\right|^{2}\right]+\frac{1}{4}\left(1-\sum_{i=1}^{m} u_{i}^{2}\right)^{2}\right\} d x^{\prime} \equiv C .
$$

For the rest of this section we study monotonicity formulae for solutions of system (1.15). Consider the following function $I_{\alpha}(r)$ for any $r>0$

$$
I_{\alpha}(r):=\frac{1}{r^{n-\alpha}} \int_{B_{r}}\left[\sum_{i=1}^{m} \Phi\left(\left|\nabla u_{i}\right|^{2}\right)-2 \tilde{H}(u)\right] .
$$

For the case of scalar equation, that is when $m=1$, the following monotonicity formula holds. Note that this is a direct consequence of Theorem A.

Theorem B. [17] Suppose that $m=1$ and $u$ is a solution of [1.11). In addition, suppose that assumptions of Theorem $A$ hold. Then the functional $I_{\alpha}(r)$ when $\alpha \geq 1$ is a monotone nondecreasing function of $r$.

For the case of system of equations, that is when $m \geq 2$, we provide a weaker version of the monotonicity formula provided in Theorem B, under certain lower bounds on $\alpha$ depending on $\Phi$.

Theorem 2.2. Let $u=\left(u_{i}\right)_{i=1}^{m}$ be a solution of (1.15) when $m \geq 2$ and $\tilde{H}(u) \leq 0$. Suppose that there exists a constant $\alpha$ such that

$$
\alpha \geq \alpha^{*}:=\inf _{s>0}\left\{\frac{2 s \Phi^{\prime}(s)}{\Phi(s)}\right\} .
$$

Then, the functional $I_{\alpha}(r)$ is a monotone nondecreasing function of $r$. In particular,

$$
I_{\alpha}^{\prime}(r) \geq \frac{2}{r^{n-\alpha}} \int_{\partial B_{r}} \sum_{i=1}^{m} \Phi^{\prime}\left(\left|\nabla u_{i}\right|^{2}\right)\left(\partial_{r} u_{i}\right)^{2}-\frac{2 \alpha}{r^{n-\alpha+1}} \int_{B_{r}} \tilde{H}(u) .
$$


Unlike Theorem B, conditions (A) and (B) do not appear in assumptions of the above theorem for the case of system of equations. This implies that Theorem 2.2 is valid for a larger class of nonlinearities $\Phi$ compared to Theorem B. However, as mentioned in Section 1 the constant $\alpha^{*}$ must be greater than one, due to assumptions on $\Phi$. For the sake of convenience of readers we clarify this here as well. Consider the auxiliary function $h(s):=-2 \Phi^{\prime}(s) s+\alpha \Phi(s)$ regarding terms appeared in (2.16). Note that from assumptions on $\Phi$, i.e. $2 s \Phi^{\prime \prime}(s)+\Phi^{\prime}(s)>0$ when $s>0$ and $h(0)=\alpha \Phi(0)=0$ one can see that $h^{\prime}(s)=-\left[2 s \Phi^{\prime \prime}(s)+\right.$ $\left.\Phi^{\prime}(s)\right]+(\alpha-1) \Phi^{\prime}(s)$ is negative when $\alpha \leq 1$. We now compute $\alpha^{*}$, provided in (2.16) , for various choices of $\Phi$.

Corollary 2.2. Suppose that $m \geq 2$ and $\tilde{H} \leq 0$. Consider the following particular functions $\Phi$.

(i) Let $\Phi(s)=s$ and $u=\left(u_{i}\right)_{i=1}^{m}$ be a solution of the semilinear system of equations

$$
-\Delta u_{i}=H_{i}(u) \text { in } \mathbb{R}^{n} \text {. }
$$

Then for all $\alpha \geq \alpha^{*}=2$, the function $I_{\alpha}(r)$ is monotone nondecreasing in $r$.

(ii) Let $\Phi(s)=2(\sqrt{1+s}-1)$ and $u=\left(u_{i}\right)_{i=1}^{m}$ be a solution of the mean curvature system of equations

$$
-\operatorname{div}\left(\frac{\nabla u_{i}}{\sqrt{1+\left|\nabla u_{i}\right|^{2}}}\right)=H_{i}(u) \text { in } \mathbb{R}^{n} .
$$

Then for all $\alpha \geq \alpha^{*}=2$, the function $I_{\alpha}(r)$ is monotone nondecreasing in $r$.

(iii) Let $\Phi(s)=\frac{2}{p} s^{\frac{p}{2}}$ and $u=\left(u_{i}\right)_{i=1}^{m}$ be a solution of $p$-Laplacian system of equations

$$
-\operatorname{div}\left(\left|\nabla u_{i}\right|^{p-2} \nabla u_{i}\right)=H_{i}(u) \text { in } \mathbb{R}^{n} .
$$

Then for all $\alpha \geq \alpha^{*}=p$, the function $I_{\alpha}(r)$ is monotone nondecreasing in $r$.

To provide a proof for Theorem 2.2 we present a few technical estimates. We follow a classical argument regarding Pohozaev and Rellich type identities [46] to prove the following identity on a ball of radius $r$.

Lemma 2.1. Suppose that $u=\left(u_{i}\right)$ is a solution of (1.15) then

$$
\begin{aligned}
-n \int_{B_{r}} \sum_{i=1}^{m} \Phi\left(\left|\nabla u_{i}\right|^{2}\right)= & 2 r \int_{\partial B_{r}} \sum_{i=1}^{m} \Phi^{\prime}\left(\left|\nabla u_{i}\right|^{2}\right)\left(\partial_{r} u_{i}\right)^{2}-r \int_{\partial B_{r}} \sum_{i=1}^{m} \Phi\left(\left|\nabla u_{i}\right|^{2}\right) \\
& -2 \int_{B_{r}} \sum_{i=1}^{m} \Phi^{\prime}\left(\left|\nabla u_{i}\right|^{2}\right)\left|\nabla u_{i}\right|^{2}-2 n \int_{B_{r}} H(u)+2 r \int_{B_{r}} H(u) .
\end{aligned}
$$

Proof. Multiply the $i^{\text {th }}$ equation of (1.15) with $x \cdot \nabla u_{i}$ and then apply the divergence theorem to get

$$
\begin{aligned}
\int_{\partial B_{r}} \Phi\left(\left|\nabla u_{i}\right|^{2}\right)= & 2 \int_{\partial B_{r}} \Phi^{\prime}\left(\left|\nabla u_{i}\right|^{2}\right)\left(\partial_{r} u_{i}\right)^{2}-\frac{2}{r} \int_{B_{r}} \Phi^{\prime}\left(\left|\nabla u_{i}\right|^{2}\right)\left|\nabla u_{i}\right|^{2} \\
& -\frac{2}{r} \int_{B_{r}}\left(x \cdot \nabla u_{i}\right) \operatorname{div}\left(\Phi^{\prime}\left(\left|\nabla u_{i}\right|^{2}\right) \nabla u_{i}\right)+\frac{n}{r} \int_{B_{r}} \Phi\left(\left|\nabla u_{i}\right|^{2}\right),
\end{aligned}
$$

for each $i=1, \cdots, m$. Doing some straightforward computations as well as applying (1.15) gives the desired result.

We are now ready to provide a proof for Theorem 2.2 .

Proof of Theorem 2.2 Differentiating $I(r)$, given by (2.15), with respect to $r$ gives

$$
\begin{aligned}
I_{\alpha}^{\prime}(r) r^{n-\alpha+1}= & (\alpha-n) \int_{B_{r}} \sum_{i=1}^{m} \Phi\left(\left|\nabla u_{i}\right|^{2}\right)-2 \tilde{H}(u) \\
& +r \int_{\partial B_{r}} \sum_{\substack{i=1 \\
m}} \Phi\left(\left|\nabla u_{i}\right|^{2}\right)-2 \tilde{H}(u) .
\end{aligned}
$$


Substituting the value of $-n \int_{B_{r}} \sum_{i=1}^{m} \Phi\left(\left|\nabla u_{i}\right|^{2}\right)$, as it is provided in Lemma 2.1, one can show that

$$
\begin{aligned}
I_{\alpha}^{\prime}(r) r^{n-\alpha+1}= & 2 r \int_{\partial B_{r}} \sum_{i=1}^{m} \Phi^{\prime}\left(\left|\nabla u_{i}\right|^{2}\right)\left(\partial_{r} u_{i}\right)^{2} \\
& +\int_{B_{r}}\left[\sum_{i=1}^{m}\left(-2 \Phi^{\prime}\left(\left|\nabla u_{i}\right|^{2}\right)\left|\nabla u_{i}\right|^{2}+\alpha \Phi\left(\left|\nabla u_{i}\right|^{2}\right)\right)-2 \alpha \tilde{H}(u)\right] .
\end{aligned}
$$

The rest of the proof is straightforward.

As an immediate consequence of Theorem 2.2 we have the following Liouville theorem for solutions of (1.15) with a finite energy.

Theorem 2.3. Suppose that assumptions of Theorem 2.2 hold. Assume also that $u=\left(u_{i}\right)_{i=1}^{m}$ has a finite energy that is

$$
\int_{\mathbb{R}^{n}}\left[\sum_{i=1}^{m} \Phi\left(\left|\nabla u_{i}\right|^{2}\right)-2 \tilde{H}(u)\right] d x<\infty
$$

Then each $u_{i}$ must be constant in dimensions $n \geq \alpha$ for $i=1, \cdots, m$.

Proof. First suppose that $n>\alpha$. From Theorem 2.2 for any $R>r$ we have

$$
\begin{aligned}
0 \leq I_{\alpha}(r) & =\frac{1}{r^{n-\alpha}} \int_{B_{r}}\left[\sum_{i=1}^{m} \Phi\left(\left|\nabla u_{i}\right|^{2}\right)-2 \tilde{H}(u)\right] \\
& \leq \frac{1}{R^{n-\alpha}} \int_{B_{R}}\left[\sum_{i=1}^{m} \Phi\left(\left|\nabla u_{i}\right|^{2}\right)-2 \tilde{H}(u)\right] \\
& \leq \frac{1}{R^{n-\alpha}} \int_{\mathbb{R}^{n}}\left[\sum_{i=1}^{m} \Phi\left(\left|\nabla u_{i}\right|^{2}\right)-2 \tilde{H}(u)\right] .
\end{aligned}
$$

Sending $R \rightarrow \infty$, we get the desired result. Now suppose that $n=\alpha$. Again from Theorem 2.2 we have

$$
r I_{\alpha}^{\prime}(r) \geq-2 \alpha \int_{B_{r}} \tilde{H}(u)
$$

From this for any $r>\bar{r}$, where $\bar{r}$ is fixed, we have

$$
I_{\alpha}(r) \geq I_{\alpha}(\bar{r})-2 \alpha \ln \left(\frac{r}{\bar{r}}\right) \int_{B_{\bar{r}}} \tilde{H}(u) .
$$

Note that (2.21) implies that $\lim _{r \rightarrow \infty} I_{\alpha}(r)<\infty$. From this and (2.23) we conclude that $\tilde{H}=0$. The fact that each component $u_{i}$ is harmonic together with (2.21) completes the proof.

Another consequence of the monotonicity formula, given in (2.17), is the following lower bound on the energy.

Corollary 2.3. Suppose that assumptions of Theorem 2.2 hold. Then, the following lower bound holds for the energy functional

$$
\int_{B_{R}}\left[\sum_{i=1}^{m} \Phi\left(\left|\nabla u_{i}\right|^{2}\right)-2 \tilde{H}(u)\right] d x \geq C R^{\alpha-n} \quad \text { for all } R>1,
$$

where $C=I(1)$ is independent from $R$.

As the next theorem, we prove an upper bound on the energy function. 
Theorem 2.4. Suppose that $u=\left(u_{i}\right)_{i=1}^{m}$ is a bounded $H$-monotone solution of (1.15) such that for each $i=1, . ., m$,

$$
\lim _{x_{n} \rightarrow \infty} u_{i}\left(x^{\prime}, x_{n}\right)=a_{i}, \quad \forall x=\left(x^{\prime}, x_{n}\right) \in \mathbb{R}^{n}
$$

for some constants $a_{i}$. Then

$$
J_{R}(u):=\int_{B_{R}}\left[\sum_{i=1}^{m} \Phi\left(\left|\nabla u_{i}\right|^{2}\right)-2 \tilde{H}(u)+2 \tilde{H}(a)\right] d x \leq C R^{n-1},
$$

where $a=\left(a_{i}\right)_{i=1}^{m}$ and $C$ are independent from $R$.

Proof. Define the sequence of shift functions $u^{t}=\left(u_{i}^{t}\right)_{i=1}^{m}$ where $u_{i}^{t}(x):=u_{i}\left(x^{\prime}, x_{n}+t\right)$ for $t \in \mathbb{R}$ and $x=\left(x^{\prime}, x_{n}\right) \in \mathbb{R}^{n}$. Note that $u^{t}=\left(u_{i}^{t}\right)_{i=1}^{m}$ satisfies

$$
-\operatorname{div}\left(\Phi^{\prime}\left(\left|\nabla u_{i}^{t}\right|^{2}\right) \nabla u_{i}^{t}\right)=H_{i}\left(u^{t}\right) \text { in } \mathbb{R}^{n} .
$$

The fact that $u_{i}^{t}$ is convergent to $a_{i}$ pointwise, it is straightforward to see that

$$
\int_{B_{R}}\left(\tilde{H}\left(u^{t}\right)-\tilde{H}(a)\right) d x \rightarrow 0 \text { when } t \rightarrow \infty .
$$

On the other hand, multiply both sides of (2.27) with $u_{i}^{t}-a_{i}$ and integrate by parts to get

$$
-\int_{B_{R}} \Phi^{\prime}\left(\left|\nabla u_{i}^{t}\right|^{2}\right)\left|\nabla u_{i}^{t}\right|^{2}+\int_{\partial B_{R}} \Phi^{\prime}\left(\left|\nabla u_{i}^{t}\right|^{2}\right) \partial_{\nu} u_{i}^{t}\left(u_{i}^{t}-a_{i}\right)=-\int_{B_{R}} H_{i}\left(u^{t}\right)\left(u_{i}^{t}-a_{i}\right),
$$

Sending $t \rightarrow \infty$ implies that

$$
\int_{B_{R}} \Phi^{\prime}\left(\left|\nabla u_{i}^{t}\right|^{2}\right)\left|\nabla u_{i}^{t}\right|^{2} \rightarrow 0 .
$$

Note that due to the assumption $2 s \Phi^{\prime \prime}(s)+\Phi(s)>0$ when $s>0$ and $\Phi(0)=0$ we have $0 \leq \Phi(s) \leq 2 \Phi^{\prime}(s) s$ for any $s>0$. This implies that

$$
0 \leq \int_{B_{R}} \Phi\left(\left|\nabla u_{i}^{t}\right|^{2}\right) \leq 2 \int_{B_{R}} \Phi^{\prime}\left(\left|\nabla u_{i}^{t}\right|^{2}\right)\left|\nabla u_{i}^{t}\right|^{2} \rightarrow 0 \text { as } t \rightarrow 0 .
$$

From this and (2.28) we get

$$
\lim _{t \rightarrow \infty} J_{R}\left(u^{t}\right)=0 \text {. }
$$

We now use $J_{R}\left(u^{t}\right)$ to construct an upper bound on $J_{R}(u)$. Note that differentiating the energy functional with respect to $t$, one gets

$$
\partial_{t} J_{R}\left(u^{t}\right)=\sum_{i=1}^{m} \int_{B_{R}}\left[2 \Phi^{\prime}\left(\left|\nabla u_{i}^{t}\right|^{2}\right) \nabla u_{i}^{t} \cdot \nabla\left(\partial_{t} u_{i}^{t}\right)-2 H_{i}\left(u^{t}\right) \partial_{t} u_{i}^{t}\right] .
$$

Multiplying the system of equations (2.27) with $\partial_{t} u^{t}$ and performing integration by parts we obtain

$$
\begin{aligned}
\int_{B_{R}} H_{i}\left(u^{t}\right) \partial_{t} u_{i}^{t}= & \int_{B_{R}} \Phi^{\prime}\left(\left|\nabla u_{i}^{t}\right|^{2}\right) \nabla u_{i}^{t} \cdot \nabla\left(\partial_{t} u_{i}^{t}\right) \\
& -\int_{\partial B_{R}} \Phi^{\prime}\left(\left|\nabla u_{i}^{t}\right|^{2}\right) \partial_{\nu} u_{i}^{t} \partial_{t} u_{i}^{t}
\end{aligned}
$$

for each $i=1, \cdots, m$. From (2.32) and (2.31) we obtain

$$
\partial_{t} E_{R}\left(u^{t}\right)=\sum_{i=1}^{m} \int_{\partial B_{R}} \Phi^{\prime}\left(\left|\nabla u_{i}^{t}\right|^{2}\right) \partial_{\nu} u_{i}^{t} \partial_{t} u_{i}^{t} .
$$

Note that there exist a constant $M$ such that $-M \leq \Phi^{\prime}\left(\left|\nabla u_{i}^{t}\right|^{2}\right) \partial_{\nu} u^{t} \leq M$ and $\partial_{t} u_{i}^{t}>0>\partial_{t} u_{j}^{t}$ for $i \in \bar{I}$ and $j \in \bar{J}$. Therefore,

$$
\partial_{t} E_{R}\left(u^{t}\right) \geq M \int_{\partial B_{R}}\left(\sum_{j \in \bar{J}} \partial_{t} u_{j}^{t}-\sum_{i \in \bar{I}} \partial_{t} u_{i}^{t}\right) d S .
$$


Therefore,

$$
\begin{aligned}
J_{R}(u) & =J_{R}\left(u^{t}\right)-\int_{0}^{t} \partial_{t} J_{R}\left(u^{s}\right) d s \\
& \leq J_{R}\left(u^{t}\right)+M \int_{0}^{t} \int_{\partial B_{R}}\left(\sum_{i \in \bar{I}} \partial_{s} u_{i}^{s}-\sum_{j \in \bar{J}} \partial_{s} u_{j}^{s}\right) d S d s \\
& =J_{R}\left(u^{t}\right)+M \int_{\partial B_{R}}\left(\sum_{i \in \bar{I}}\left(u_{i}^{t}-u_{i}\right)+\sum_{j \in \bar{J}}\left(u_{j}-u_{j}^{t}\right)\right) d S .
\end{aligned}
$$

From the definiton of $H$-monotonicity and the sets of $\bar{I}, \bar{J}$ we have $u_{i}<u_{i}^{t}$ and $u_{j}^{t}<u_{j}$ for all $i \in \bar{I}, j \in \bar{J}$ and $t \in \mathbb{R}^{+}$. Therefore,

$$
J_{R}(u) \leq J_{R}\left(u^{t}\right)+M \int_{\partial B_{R}}\left(\sum_{i \in I}\left(u_{i}^{t}-u_{i}\right)+\sum_{j \in J}\left(u_{j}-u_{j}^{t}\right)\right) d S \text { for all } t \in \mathbb{R}^{+},
$$

where $M:=\max _{i=1}^{m}\left\{|| \Phi^{\prime}\left(\left|\nabla u_{i}\right|^{2}\right)\left|\nabla u_{i}\right| \|_{L^{\infty}\left(\mathbb{R}^{n}\right)}\right\}$. The upper bound (2.36) implies

$$
E_{R}(u) \leq E_{R}\left(u^{t}\right)+C\left|\partial B_{R}\right| \text { for all } t \in \mathbb{R}^{+} .
$$

Sending $t \rightarrow \infty$ and using (2.30), finally we obtain that

$$
J_{R}(u) \leq C\left|\partial B_{R}\right| \leq C R^{n-1} .
$$

This provides the desired result.

Before we finish this section, let us mention a couple of open problems for the system of equations (1.15).

Open Problem 1. Under what assumptions on $H=\left(H_{i}\right)_{i=1}^{m}$ and solutions, one can provide a counterpart of the pointwise inequalities provided by Modica in [42] and Caffarelli et al. in [17] for solutions of [1.15) when $m \geq 2$ ?

Open Problem 2. In the light of Theorem 2.4. Corollary 2.3 and Theorem B, one might expect that $I_{\alpha}(r)$ should be a nondecreasing function of $r$ when $\alpha \geq \alpha^{*}=1$ for the case of systems that is when $m \geq 2$.

\section{Geometric Poincaré and stability inequalities for systems}

Note that the matrix $\mathcal{A}(\eta):=\left(a_{i, j}(\eta)\right)_{i, j=1}^{n}$ where $a_{i, j}(\eta)$ is defined by

$$
a_{i, j}(\eta):=2 \Phi^{\prime \prime}\left(|\eta|^{2}\right) \eta_{i} \eta_{j}+\Phi^{\prime}\left(|\eta|^{2}\right) \delta_{i j} .
$$

is symmetric and positive definite for every $\eta \in \mathbb{R}^{n}$. This is because for any $\zeta \in \mathbb{R}^{n}$,

$$
\begin{aligned}
\mathcal{A}(\eta) \zeta . \zeta & =\sum_{i, j=1}^{m} 2 \zeta_{i} \zeta_{j} \Phi^{\prime \prime}\left(|\eta|^{2}\right) \eta_{i} \eta_{j}+\Phi^{\prime}\left(|\eta|^{2}\right) \zeta_{i} \zeta_{j} \delta_{i, j} \\
& =2 \Phi^{\prime \prime}\left(|\eta|^{2}\right)|\zeta \cdot \eta|^{2}+\Phi^{\prime}\left(|\eta|^{2}\right)|\zeta|^{2} .
\end{aligned}
$$

Note that when $\Phi^{\prime \prime}(\eta)$ is positive clearly $A(\eta) \zeta . \zeta$ is positive since $\Phi^{\prime}$ is positive and when $\Phi^{\prime \prime}(\eta)$ is negative applying Young's inequality together with $2 \Phi^{\prime \prime}(s) s+\Phi^{\prime}(s)>0$ when $s>0$ implies that $A(\eta) \zeta . \zeta$ is positive. We are now ready to prove the stability inequality for solutions of (1.15). Note that such an inequality for the case of semilinear systems is given in [20, 34, 35].

Lemma 3.1. Let $u=\left(u_{i}\right)_{i=1}^{m}$ denote a stable solution of (1.15). Then

$$
\sum_{i, j=1}^{m} \int_{\mathbb{R}^{n}} \sqrt{\partial_{j} H_{i}(u) \partial_{i} H_{j}(u)} \zeta_{i} \zeta_{j} \leq \sum_{i=1}^{m} \int_{\mathbb{R}^{n}} \mathcal{A}\left(\nabla u_{i}\right) \nabla \zeta_{i} \cdot \nabla \zeta_{i},
$$

for any $\zeta=\left(\zeta_{i}\right)_{i=1}^{m}$ where $\zeta_{i} \in C_{c}^{1}\left(\mathbb{R}^{n}\right)$ for $1 \leq i \leq m$. 
Proof. Since $u$ is a stable solutions, there exists a sequence $\phi=\left(\phi_{i}\right)_{i}^{m}$ that satisfies (1.26). Consider a test function $\zeta=\left(\zeta_{i}\right)_{i}^{m}$ where $\zeta_{i} \in L^{\infty}\left(\mathbb{R}^{n}\right) \cap H^{1}\left(\mathbb{R}^{n}\right)$ with compact support and multiply both sides of (1.26) with $\frac{\zeta_{i}^{2}}{\phi_{i}}$. Integrating by parts we get

$$
I_{i}:=\sum_{j=1}^{m} \int_{\mathbb{R}^{n}} \partial_{j} H_{i}(u) \phi_{j} \frac{\zeta_{i}^{2}}{\phi_{i}}=\int_{\mathbb{R}^{n}} \mathcal{A}\left(\nabla u_{i}\right) \nabla \phi_{i} \cdot\left(2 \nabla \zeta_{i} \frac{\zeta_{i}}{\phi_{i}}-\nabla \phi_{i} \frac{\zeta_{i}^{2}}{\phi_{i}^{2}}\right)=: J_{i}
$$

The fact that $\mathcal{A}\left(\nabla u_{i}\right)$ is positive definite we get

$$
\begin{aligned}
0 & \leq \mathcal{A}\left(\nabla u_{i}\right)\left(\phi_{i} \nabla \zeta_{i}-\zeta_{i} \nabla \phi_{i}\right) \cdot\left(\phi_{i} \nabla \zeta_{i}-\zeta_{i} \nabla \phi_{i}\right) \\
& =\phi_{i}^{2} \mathcal{A}\left(\nabla u_{i}\right) \nabla \zeta_{i} \cdot \nabla \zeta_{i}+\zeta_{i}^{2} \mathcal{A}\left(\nabla u_{i}\right) \nabla \phi_{i} \cdot \nabla \phi_{i}-\zeta_{i} \phi_{i} \mathcal{A}\left(\nabla u_{i}\right) \nabla \phi_{i} \cdot \nabla \zeta_{i}
\end{aligned}
$$

Applying this to (3.4) for each $i$ we obtain

$$
J_{i} \leq \int_{\mathbb{R}^{n}} \mathcal{A}\left(\nabla u_{i}\right) \nabla \zeta_{i} \cdot \nabla \zeta_{i}
$$

For the left-hand side we have,

$$
\begin{aligned}
\sum_{i=1}^{m} I_{i} & =\sum_{i, j=1}^{m} \int_{\mathbb{R}^{n}} \partial_{j} H_{i}(u) \phi_{j} \frac{\zeta_{i}^{2}}{\phi_{i}} \\
& =\sum_{i<j}^{m} \int_{\mathbb{R}^{n}} \partial_{j} H_{i}(u) \phi_{j} \frac{\zeta_{i}^{2}}{\phi_{i}}+\sum_{i>j}^{n} \int_{\mathbb{R}^{n}} \partial_{j} H_{i}(u) \phi_{j} \frac{\zeta_{i}^{2}}{\phi_{i}}+\sum_{i=1}^{m} \int_{\mathbb{R}^{n}} \partial_{i} H_{i}(u) \zeta_{i}^{2} \\
& =\sum_{i<j}^{m} \int_{\mathbb{R}^{n}} \partial_{j} H_{i}(u) \phi_{j} \frac{\zeta_{i}^{2}}{\phi_{i}}+\sum_{i<j}^{m} \int_{\mathbb{R}^{n}} \partial_{i} H_{j}(u) \phi_{i} \frac{\zeta_{j}^{2}}{\phi_{j}}+\sum_{i=1}^{m} \int_{\mathbb{R}^{n}} \partial_{i} H_{i}(u) \zeta_{i}^{2} \\
& =\sum_{i<j}^{m} \int_{\mathbb{R}^{n}}\left(\partial_{j} H_{i}(u) \phi_{j} \frac{\zeta_{i}^{2}}{\phi_{i}}+\partial_{i} H_{j}(u) \phi_{i} \frac{\zeta_{j}^{2}}{\phi_{j}}\right)+\sum_{i=1}^{m} \int_{\mathbb{R}^{n}} \partial_{i} H_{i}(u) \zeta_{i}^{2} \\
& \geq 2 \sum_{i<j}^{m} \int_{\mathbb{R}^{n}} \sqrt{\partial_{j} H_{i}(u) \partial_{i} H_{j}(u)} \zeta_{i} \zeta_{j}+\sum_{i=1}^{m} \int_{\mathbb{R}^{n}} \partial_{i} H_{i}(u) \zeta_{i}^{2} \\
& =\sum_{i, j=1}^{m} \int_{\mathbb{R}^{n}} \sqrt{\partial_{j} H_{i}(u) \partial_{i} H_{j}(u)} \zeta_{i} \zeta_{j} .
\end{aligned}
$$

This finishes the proof.

We now apply the stability inequality to provide a geometric Poincaré inequality of the following from. For the case of scalar equations that is when $m=1$ this inequality was driven by Sternberg-Zumbrun in [53] and it was applied in this context by Farina-Sciunzi-Valdinoci [33] and references therein to provide De Giorgi type results. Note also that Cabré applied this type inequality to prove regularity of extremal solutions of nonlinear eigenvalue problems in [13]. For the case of system of equations that is when $m \geq 1$ this inequality was first proved by Ghoussoub and the author in [35] and they applied the inequality to conclude De Giorgi type results for system of equations. Let us mention that interested readers can find similar geometric Poincaré inequalities in these references as well [25, 26, 31, 52]. 
Theorem 3.1. Assume that $m, n \geq 1$ and $u=\left(u_{i}\right)_{i=1}^{m}$ is a stable solution of (1.15). Then, for any $\eta=\left(\eta_{k}\right)_{k=1}^{m} \in C_{c}^{1}\left(\mathbb{R}^{n}\right)$, the following inequality holds;

$$
\begin{aligned}
& \sum_{i \neq j} \int_{\mathbb{R}^{n}}\left[\sqrt{\partial_{j} H_{i}(u) \partial_{i} H_{j}(u)}\left|\nabla u_{i}\right|\left|\nabla u_{j}\right| \eta_{i} \eta_{j}-\partial_{j} H_{i}(u) \nabla u_{i} \cdot \nabla u_{j} \eta_{i}^{2}\right] \\
& +\sum_{i=1}^{m} \int_{\left\{\left|\nabla u_{i}\right| \neq 0\right\} \cap \mathbb{R}^{n}} \Phi^{\prime}\left(\left|\nabla u_{i}\right|^{2}\right)\left|\nabla u_{i}\right|^{2} \mathcal{K}_{i}^{2} \eta_{i}^{2} \\
& \quad+\sum_{i=1}^{m} \int_{\left\{\left|\nabla u_{i}\right| \neq 0\right\} \cap \mathbb{R}^{n}}\left[2 \Phi^{\prime \prime}\left(\left|\nabla u_{i}\right|^{2}\right)\left|\nabla u_{i}^{2}\right|+\Phi^{\prime}\left(\left|\nabla u_{i}\right|^{2}\right)\right]\left|\nabla T_{i}\right| \nabla u_{i}||^{2} \eta_{i}^{2} \\
& \leq \sum_{i=1}^{m} \int_{\mathbb{R}^{n}} 2\left|\nabla u_{i}\right|^{2} \Phi^{\prime \prime}\left(\left|\nabla u_{i}\right|^{2}\right)\left|\nabla u_{i} \cdot \nabla \eta_{i}\right|^{2}+\Phi^{\prime}\left(\left|\nabla u_{i}\right|^{2}\right)\left|\nabla u_{i}\right|^{2}\left|\nabla \eta_{i}\right|^{2},
\end{aligned}
$$

where $\nabla_{T_{i}}$ stands for the tangential gradient along a given level set of $u_{i}$ and $\mathcal{K}_{i}^{2}$ for the sum of squares of principal curvatures of such a level set.

Proof. Suppose that $\eta=\left(\eta_{1}, \ldots, \eta_{m}\right)$ for $\eta_{i} \in C_{c}^{1}\left(\mathbb{R}^{n}\right)$ is a test function. Test the stability inequality (5.8) with $\zeta_{i}=\left|\nabla u_{i}\right| \eta_{i}$ to get

$$
\begin{aligned}
\sum_{i=1}^{m} \int_{\mathbb{R}^{n}} \partial_{i} H_{i}(u)\left|\nabla u_{i}\right|^{2} \eta_{i}^{2} \leq & -\sum_{i \neq j} \int_{\mathbb{R}^{n}} \sqrt{\partial_{j} H_{i}(u) \partial_{i} H_{j}(u)}\left|\nabla u_{i}\right|\left|\nabla u_{j}\right| \eta_{i} \eta_{j} \\
& +\sum_{i=1}^{m} \int_{\mathbb{R}^{n}}\left|\nabla u_{i}\right|^{2} \mathcal{A}\left(\nabla u_{i}\right) \nabla \eta_{i} \cdot \nabla \eta_{i} \\
& +\sum_{i=1}^{m} \int_{\mathbb{R}^{n}} \eta_{i}^{2} \mathcal{A}\left(\nabla u_{i}\right) \nabla\left|\nabla u_{i}\right| \cdot \nabla\left|\nabla u_{i}\right| \\
& +\frac{1}{2} \sum_{i=1}^{m} \int_{\mathbb{R}^{n}} \mathcal{A}\left(\nabla u_{i}\right) \nabla\left|\nabla u_{i}\right|^{2} \cdot \nabla \eta_{i}^{2} .
\end{aligned}
$$

Straightforward calculations show that for each $k$,

$$
\partial_{x_{k}}\left(\Phi^{\prime}\left(\left|\nabla u_{i}\right|^{2}\right) \nabla u_{i}\right)=\mathcal{A}\left(\nabla u_{i}\right) \nabla \partial_{x_{k}} u_{i}
$$

Applying this and differentiating the $i^{\text {th }}$ equation of (1.15) with respect to $x_{k}$ for each $i=1,2, \ldots, m$ we get

$$
-\operatorname{div}\left(\mathcal{A}\left(\nabla u_{i}\right) \nabla \partial_{x_{k}} u_{i}\right)=\sum_{j=1}^{m} \partial_{j} H_{i}(u) \partial_{x_{k}} u_{i} .
$$

Multiplying the above with $\eta_{i}^{2} \partial_{k} u_{i}$, integrating by parts and taking sum on the indices $i, k$ we get

$$
\begin{aligned}
& \sum_{i=1}^{m} \int_{\mathbb{R}^{n}} \partial_{i} H_{i}(u)\left|\nabla u_{i}\right|^{2} \eta_{i}^{2} \\
= & -\sum_{j \neq i} \int_{\mathbb{R}^{n}} \partial_{j} H_{i}(u) \nabla u_{i} \cdot \nabla u_{j} \eta_{i}^{2}+\sum_{i=1}^{m} \sum_{k=1}^{n} \int_{\mathbb{R}^{n}} \mathcal{A}\left(\nabla u_{i}\right) \nabla\left(\partial_{x_{k}} u_{i}\right) \cdot \nabla\left(\partial_{x_{k}} \eta_{i}^{2}\right) \\
= & -\sum_{j \neq i} \int_{\mathbb{R}^{n}} \partial_{j} H_{i}(u) \nabla u_{i} \cdot \nabla u_{j} \eta_{i}^{2}+\sum_{i=1}^{m} \sum_{k=1}^{n} \int_{\mathbb{R}^{n}} \eta_{i}^{2} \mathcal{A}\left(\nabla u_{i}\right) \nabla\left(\partial_{x_{k}} u_{i}\right) \cdot \nabla\left(\partial_{x_{k}} u_{i}\right) \\
& +\frac{1}{2} \sum_{i=1}^{m} \int_{\mathbb{R}^{n}} \mathcal{A}\left(\nabla u_{i}\right) \nabla\left|\nabla u_{i}\right|^{2} \cdot \nabla \eta_{i}^{2} .
\end{aligned}
$$


Equating (3.10) and (3.7) we get the following since the term $\frac{1}{2} \sum_{k=1}^{m} \int_{\mathbb{R}^{n}} \mathcal{A}\left(\nabla u_{i}\right) \nabla\left|\nabla u_{i}\right|^{2} \cdot \nabla \eta_{i}^{2}$ cancels out,

$$
\begin{aligned}
& \sum_{i \neq j} \int_{\mathbb{R}^{n}}\left[\sqrt{\partial_{j} H_{i}(u) \partial_{i} H_{j}(u)}\left|\nabla u_{i}\right|\left|\nabla u_{j}\right| \eta_{i} \eta_{j}-\partial_{j} H_{i}(u) \nabla u_{i} \cdot \nabla u_{j} \eta_{i}^{2}\right] \\
& +\sum_{i, k=1}^{m} \int_{\mathbb{R}^{n}} \mathcal{A}\left(\nabla u_{i}\right) \nabla\left(\partial_{x_{k}} u_{i}\right) \cdot \nabla\left(\partial_{x_{k}} u_{i}\right) \eta_{i}^{2} \\
& -\sum_{i=1}^{m} \int_{\mathbb{R}^{n}} \mathcal{A}\left(\nabla u_{i}\right) \nabla\left|\nabla u_{i}\right| \cdot \nabla\left|\nabla u_{i}\right| \eta_{i}^{2} \\
\leq & \sum_{i=1}^{m} \int_{\mathbb{R}^{n}}\left|\nabla u_{i}\right|^{2} \mathcal{A}\left(\nabla u_{i}\right) \nabla \eta_{i} \cdot \nabla \eta_{i} .
\end{aligned}
$$

For the rest of the proof, we simplify two terms (3.12) and (3.13) in the left-hand side of the above inequality. From the definition of there matrix $\mathcal{A}$, in the light of (3.2), we get

$$
\begin{aligned}
\sum_{k=1}^{n} \mathcal{A}\left(\nabla u_{i}\right) \nabla\left(\partial_{x_{k}} u_{i}\right) \cdot \nabla\left(\partial_{x_{k}} u_{i}\right)= & 2 \Phi^{\prime \prime}\left(\left|\nabla u_{i}\right|^{2}\right) \sum_{k=1}^{n}\left|\nabla u_{i} \cdot \nabla \partial_{x_{k}} u_{i}\right|^{2} \\
& +\Phi^{\prime}\left(\left|\nabla u_{i}\right|^{2}\right) \sum_{k=1}^{n}\left|\nabla \partial_{x_{k}} u_{i}\right|^{2} .
\end{aligned}
$$

Straightforward calculations show that

$$
\sum_{k=1}^{n}\left|\nabla u_{i} \cdot \nabla \partial_{x_{k}} u_{i}\right|^{2}=\left.\left.\frac{1}{4}|\nabla| \nabla u_{i}\right|^{2}\right|^{2}=\left.\left|\nabla u_{i}\right|^{2}|\nabla| \nabla u_{i}\right|^{2} .
$$

From (3.15) and (3.16) we obtain the following form for the term in (3.12)

$$
\begin{aligned}
& \sum_{i=1}^{m} \sum_{k=1}^{n} \int_{\mathbb{R}^{n}} \mathcal{A}\left(\nabla u_{i}\right) \nabla\left(\partial_{x_{k}} u_{i}\right) \cdot \nabla\left(\partial_{x_{k}} u_{i}\right) \eta_{i}^{2} \\
= & \left.\sum_{i=1}^{m} \int_{\mathbb{R}^{n}} 2\left|\nabla u_{i}\right|^{2} \Phi^{\prime \prime}\left(\left|\nabla u_{i}\right|^{2}\right)|\nabla| \nabla u_{i}\right|^{2} \eta_{i}^{2} \\
& +\sum_{i=1}^{m} \int_{\mathbb{R}^{n}} \Phi^{\prime}\left(\left|\nabla u_{i}\right|^{2}\right) \sum_{k=1}^{n}\left|\nabla \partial_{x_{k}} u_{i}\right|^{2} \eta_{i}^{2} .
\end{aligned}
$$

Similarly, from the definition of the matrix $\mathcal{A}$, i.e. using (3.2), we get

$$
\begin{aligned}
\mathcal{A}\left(\nabla u_{i}\right) \nabla\left|\nabla u_{i}\right| \cdot \nabla\left|\nabla u_{i}\right|= & 2 \Phi^{\prime \prime}\left(\left|\nabla u_{i}\right|^{2}\right)\left|\nabla u_{i} \cdot \nabla\right| \nabla u_{i}||^{2} \\
& +\left.\Phi^{\prime}\left(\left|\nabla u_{i}\right|^{2}\right)|\nabla| \nabla u_{i}\right|^{2} .
\end{aligned}
$$

This implies that the term in (3.13) is of the from

$$
\begin{aligned}
& \sum_{i=1}^{m} \int_{\mathbb{R}^{n}} \mathcal{A}\left(\nabla u_{i}\right) \nabla\left|\nabla u_{i}\right| \cdot \nabla\left|\nabla u_{i}\right| \eta_{i}^{2} \\
= & \left.\sum_{i=1}^{m} \int_{\mathbb{R}^{n}} 2 \Phi^{\prime \prime}\left(\left|\nabla u_{i}\right|^{2}\right)\left|\nabla u_{i} \cdot \nabla\right| \nabla u_{i}\right|^{2} \eta_{i}^{2} \\
& +\sum_{i=1}^{m} \int_{\mathbb{R}^{n}} \Phi^{\prime}\left(\left|\nabla u_{i}\right|^{2}\right)|\nabla| \nabla u_{i}||^{2} \eta_{i}^{2} .
\end{aligned}
$$


The difference of (3.17) and (3.15), as appeared in (3.13) and (3.12), is

$$
\begin{aligned}
& \sum_{i=1}^{m} \sum_{k=1}^{n} \int_{\mathbb{R}^{n}} \mathcal{A}\left(\nabla u_{i}\right) \nabla\left(\partial_{x_{k}} u_{i}\right) \cdot \nabla\left(\partial_{x_{k}} u_{i}\right) \eta_{i}^{2} \\
& -\sum_{i=1}^{m} \int_{\mathbb{R}^{n}} \mathcal{A}\left(\nabla u_{i}\right) \nabla\left|\nabla u_{i}\right| \cdot \nabla\left|\nabla u_{i}\right| \eta_{i}^{2} \\
= & 2 \sum_{i=1}^{m} \int_{\Omega} \eta_{i}^{2}\left|\nabla u_{i}\right|^{2}\left[\left.|\nabla| \nabla u_{i}\right|^{2}-\frac{1}{\left|\nabla u_{i}\right|^{2}}\left|\nabla u_{i} \cdot \nabla\right| \nabla u_{i}||^{2}\right] \Phi^{\prime \prime}\left(\left|\nabla u_{i}\right|^{2}\right) \\
& +\sum_{i=1}^{m} \int_{\Omega} \Phi^{\prime}\left(\left|\nabla u_{i}\right|^{2}\right)\left[\sum_{k=1}^{n}\left|\nabla \partial_{x_{k}} u_{i}\right|^{2}-|\nabla| \nabla u_{i}||^{2}\right] \eta_{i}^{2} .
\end{aligned}
$$

where $\Omega=\left\{\left|\nabla u_{i}\right| \neq 0\right\} \cap \mathbb{R}^{n}$. We now simplify (3.22) and (3.23) via applying the tangential gradient and curvatures. Suppose that $\left|\nabla u_{i}\right| \neq 0$ at a point $x \in \mathbb{R}^{n}$, then

$$
\left.\left|\nabla_{T}\right| \nabla u_{i}\right|^{2}=|\nabla| \nabla u_{i}||^{2}-\frac{1}{\left|\nabla u_{i}\right|^{2}}\left|\nabla u_{i} \cdot \nabla\right| \nabla u_{i}||^{2},
$$

where $\nabla_{T}$ denotes the orthogonal projection of the gradient along this level set. In addition, according to formula (2.1) given in [53], the following geometric identity between the tangential gradients and curvatures holds,

$$
\sum_{k=1}^{n}\left|\nabla \partial_{k} u_{i}\right|^{2}-\left.|\nabla| \nabla u_{i}\right|^{2}=\left|\nabla u_{i}\right|^{2} \mathcal{K}_{i}^{2}+\left|\nabla_{T}\right| \nabla u_{i}||^{2}
$$

for $\mathcal{K}_{i}^{2}:=\sum_{l=1}^{n-1} \kappa_{l}^{2}$ where $\kappa_{l}$ is the principal curvatures of the level set of $u_{i}$ at $x$. Substituting (3.24) and (3.25) in (3.22) and (3.23) we get

$$
\begin{aligned}
& \sum_{i=1}^{m} \sum_{k=1}^{n} \int_{\mathbb{R}^{n}} \mathcal{A}\left(\nabla u_{i}\right) \nabla\left(\partial_{x_{k}} u_{i}\right) \cdot \nabla\left(\partial_{x_{k}} u_{i}\right) \eta_{i}^{2} \\
& -\sum_{i=1}^{m} \int_{\mathbb{R}^{n}} \mathcal{A}\left(\nabla u_{i}\right) \nabla\left|\nabla u_{i}\right| \cdot \nabla\left|\nabla u_{i}\right| \eta_{i}^{2} \\
= & \sum_{i=1}^{m} \int_{\left\{\left|\nabla u_{i}\right| \neq 0\right\} \cap \mathbb{R}^{n}} 2\left|\nabla u_{i}\right|^{2} \Phi^{\prime \prime}\left(\left|\nabla u_{i}\right|^{2}\right)\left|\nabla_{T}\right| \nabla u_{i}||^{2} \eta_{i}^{2} \\
& +\sum_{i=1}^{m} \int_{\left\{\left|\nabla u_{i}\right| \neq 0\right\} \cap \mathbb{R}^{n}} \Phi^{\prime}\left(\left|\nabla u_{i}\right|^{2}\right)\left[\left|\nabla u_{i}\right|^{2}+|\nabla T| \nabla u_{i}||^{2}\right] \eta_{i}^{2} .
\end{aligned}
$$

Finally, substitution of (3.26) in (3.13) and (3.12) completes the proof.

\section{De Giorgi type ReSUlts For SyMmetric SYStems}

In this section, we provide One dimensional symmetry results for stable and $H$-monotone solutions of symmetric system (1.15) in lower dimensions with a general nonlinearity. At first let us fix a few notations. Throughout this section we suppose that $\zeta=\left(\zeta_{i}\right)_{i=1}^{m}$ is a sequence of test functions where $\zeta_{i} \in C_{c}^{2}\left(\mathbb{R}^{n}\right) \cap[0,1]$ where $\zeta_{i} \equiv 1$ in $B_{1}$ and $\zeta_{i} \equiv 0$ in $\mathbb{R}^{n} \backslash B_{2}$. Set ${ }_{R} \zeta_{i}(x):=\zeta_{i}\left(\frac{x}{R}\right)$ and ${ }_{R} \Gamma=\left({ }_{R} \Gamma_{i}\right)_{i=1}^{m}$ where ${ }_{R} \Gamma_{i}(x):=\nabla \zeta_{i}\left(\frac{x}{R}\right)$ for any $R>1$. Note that $\left\|{ }_{R} \Gamma_{i}(x)\right\|_{L^{\infty}\left(\mathbb{R}^{n}\right)} \leq C$ where $C$ is independent from $R$ and $\nabla_{R} \zeta_{i}(x)=R^{-1}{ }_{R} \Gamma_{i}(x)$.

To set up a Liouville theorem for the quotient of partial derivatives of solutions of (1.15), we first state the following technical lemma. 
Lemma 4.1. Suppose that $u=\left(u_{i}\right)_{i=1}^{m}$ is a $H$-monotone solution of (1.15). Set $\phi_{i}:=\partial_{x_{n}} u_{i}$ and $\psi_{i}:=\nabla u_{i} \cdot \eta$ where $\eta=\left(\eta^{\prime}, 0\right) \in \mathbb{R}^{n-1} \times\{0\}$ and define $\sigma_{i}:=\frac{\psi_{i}}{\phi_{i}}$. Then the sequence of functions $\sigma=\left(\sigma_{i}\right)_{i=1}^{m}$ satisfies

$$
\operatorname{div}\left[\phi_{i}^{2} \mathcal{A}\left(\nabla u_{i}\right) \nabla \sigma_{i}\right]+\sum_{j=1}^{m} \partial_{j} H_{i}(u) \phi_{i} \phi_{j}\left(\sigma_{j}-\sigma_{i}\right) \sigma_{i}=0 \text { in } \mathbb{R}^{n} .
$$

Proof. Since the proof is straightforward we omit it here.

The fact that $\sigma=\left(\sigma_{i}\right)_{i=1}^{m}$ satisfies (4.1) motivates us to provide a Liouville theorem for system (4.1). Applying Caccioppoli type arguments we establish the following Liouvlle theorem for a slightly more general setting than (4.1). Let us mention that for the case of scalar semilinear equation, $m=1$ and $\Phi(s)=s$, this type of Liouville theorem was noted by Berestycki, Caffarelli and Nirenberg in [10] and used by Ghoussoub and Gui in [37] and later by Ambrosio and Cabré in [6] to prove the De Giorgi conjecture in dimensions two and three. Also, Ghoussoub and Gui in [38] used a slightly stronger version to show that the De Giorgi's conjecture is true in dimensions four and five for a special class of solutions that satisfy an antisymmetry condition. We also refer interested readers to [9] by Barlow, Bass and Gui and to [8] by Barlow for some probability based arguments regarding this Liouvlle theorem.

For the case of scalar quasilinear equation, $m=1$ and a general $\Phi$, this Liouville theorem is provided by Farina, Sciunzi and Valdinoci in [33] and by Danielli and Garofalo in [22]. For the case of semilinear system of equations, $m \geq 1$ and $\Phi(s)=s$, we refer to [35] by Ghoussoub and the author.

Proposition 4.1. Assume that for each $i=1, \cdots, m$ functions $\left|\nabla u_{i}\right|$ and $\phi_{i}$ are locally bounded in $\mathbb{R}^{n}$ where $\phi_{i}^{2}>0$ and $\sigma_{i} \in H_{l o c}^{1}\left(\mathbb{R}^{n}\right)$. Let

$$
\sum_{i=1}^{m} \int_{B_{2 R} \backslash B_{R}} \phi_{i}^{2} \sigma_{i}^{2} \mathcal{A}\left(\nabla u_{i}\right)_{R} \Gamma_{i} \cdot{ }_{R} \Gamma_{i} \leq C R^{2},
$$

where the constant $C$ is independent from $R>1$. Let $\sigma=\left(\sigma_{i}\right)_{i=1}^{m}$ satisfy

$$
\sigma_{i} \operatorname{div}\left[\phi_{i}^{2} \mathcal{A}\left(\nabla u_{i}\right) \nabla \sigma_{i}\right]+\sum_{j=1}^{m} h_{i j}(x) f\left(\sigma_{j}-\sigma_{i}\right) \sigma_{i} \geq 0 \quad \text { in } \mathbb{R}^{n},
$$

where $0 \leq h_{i j} \in L_{\text {loc }}^{1}\left(\mathbb{R}^{n}\right), h_{i j}=h_{j i}$ and $f \in L_{\text {loc }}^{1}(\mathbb{R})$ is an odd function such that $f(s) \geq 0$ for $s \in \mathbb{R}^{+}$. Then, each function $\sigma_{i}$ is constant for all $i=1, \ldots, m$.

Proof. The proof is strongly motived by the ideas and methods used in $[2,[6,33,35]$. Note that

$$
\begin{aligned}
\sum_{i, j=1}^{m} h_{i j}(x) \sigma_{i} f\left(\sigma_{j}-\sigma_{i}\right) & =\sum_{i<j} h_{i j} \sigma_{i} f\left(\sigma_{j}-\sigma_{i}\right)+\sum_{i>j} h_{i j} \sigma_{i} f\left(\sigma_{j}-\sigma_{i}\right) \\
& =\sum_{i<j} h_{i j} \sigma_{i} f\left(\sigma_{j}-\sigma_{i}\right)+\sum_{i<j} h_{i j} \sigma_{j} f\left(\sigma_{i}-\sigma_{j}\right) \text { since } h_{i j}=h_{j i} \\
& =-\sum_{i<j} h_{i j}\left(\sigma_{j}-\sigma_{i}\right) f\left(\sigma_{j}-\sigma_{i}\right) \text { since } f \text { is odd. } \\
& \leq 0 \text { since } h_{i j} \geq 0 \text { and } s f(s) \geq 0 \text { for any } s \in \mathbb{R} .
\end{aligned}
$$

Multiply (4.3) with a text function ${ }_{R} \zeta_{i}^{2}$ and perform integration by parts to get

$$
\sum_{i=1}^{m} \int_{\mathbb{R}^{n}} \phi_{i}^{2} \mathcal{A}\left(\nabla u_{i}\right) \nabla\left(\sigma_{i R} \zeta_{i}^{2}\right) \cdot \nabla \sigma_{i} \leq \sum_{i, j=1}^{m} \int_{\mathbb{R}^{n}} h_{i j}(x) \sigma_{i} f\left(\sigma_{j}-\sigma_{i}\right) \leq 0 .
$$

Therefore,

$$
\sum_{i=1}^{m} \int_{\mathbb{R}^{n}} \phi_{i R}^{2} \zeta_{i}^{2} \mathcal{A}\left(\nabla u_{i}\right) \nabla \sigma_{i} \cdot \nabla \sigma_{i} \leq-2 \sum_{i=1}^{m} \int_{B_{2 R} \backslash B_{R}} \phi_{i}^{2} \sigma_{i R} \zeta_{i} \mathcal{A}\left(\nabla u_{i}\right) \nabla_{R} \zeta_{i} \cdot \nabla \sigma_{i} .
$$

In the light of the Cauchy inequality with epsilon, one can see that for any $\epsilon>0$,

$$
2\left|\sigma_{i}\right|_{R} \zeta_{i}\left|\mathcal{A}\left(\nabla u_{i}\right) \nabla_{R} \zeta_{i} \cdot \nabla \sigma_{i}\right| \leq \epsilon_{R} \zeta_{i}^{2} \mathcal{A}\left(\nabla u_{i}\right) \nabla \sigma_{i} \cdot \nabla \sigma_{i}+\frac{1}{\epsilon} \sigma_{i}^{2} \mathcal{A}\left(\nabla u_{i}\right) \nabla_{R} \zeta_{i} \cdot \nabla_{R} \zeta_{i} .
$$


From this and (4.4), for every $R>1$, we get

$$
\begin{aligned}
\sum_{i=1}^{m} \int_{\mathbb{R}^{n}} \phi_{i R}^{2} \zeta_{i}^{2} \mathcal{A}\left(\nabla u_{i}\right) \nabla \sigma_{i} \cdot \nabla \sigma_{i} \leq & \epsilon \sum_{i=1}^{m} \int_{B_{2 R} \backslash B_{R}} \phi_{i R}^{2} \zeta_{i}^{2} \mathcal{A}\left(\nabla u_{i}\right) \nabla \sigma_{i} \cdot \nabla \sigma_{i} \\
& +\frac{1}{\epsilon} \sum_{i=1}^{m} \int_{B_{2 R} \backslash B_{R}} \phi_{i}^{2} \mathcal{A}\left(\nabla u_{i}\right) \nabla_{R} \zeta_{i} \cdot \nabla_{R} \zeta_{i} .
\end{aligned}
$$

From this, (4.2) and the definition of the test function ${ }_{R} \zeta_{i}$ we conclude that the following integral is bounded,

$$
\sum_{i=1}^{m} \int_{\mathbb{R}^{n}} \phi_{i}^{2} \mathcal{A}\left(\nabla u_{i}\right) \nabla \sigma_{i} \cdot \nabla \sigma_{i}<\infty .
$$

Now, sending $R \rightarrow \infty$ and $\epsilon \rightarrow \infty$ and applying (4.5) and (4.2) show that the integral in (4.6) vanishes for every $i=1, \cdots, m$. Finally, the fact that $\mathcal{A}$ is a positive definite matrix implies $\left|\nabla \sigma_{i}\right| \equiv 0$ for each $i=1, \cdots, m$. This completes the proof.

We are now ready to present the following De Giorgi type results in two dimensions.

Theorem 4.1. Suppose that $u=\left(u_{i}\right)_{i=1}^{m}$ is a classical bounded stable solution of symmetric system (1.15) in two dimensions. Assume also that $\left|\nabla u_{i}\right| \in L^{\infty}\left(\mathbb{R}^{2}\right) \cap W_{\text {loc }}^{1,2}\left(\mathbb{R}^{2}\right)$. Then each $u_{i}$ is a one dimensional function, i.e. there exists $u_{i}^{*}: \mathbb{R} \rightarrow \mathbb{R}$ and $a \in \mathbb{S}^{1}$ such that $u_{i}(x)=u_{i}^{*}(a \cdot x)$. In addition, the angle between $\nabla u_{i}$ and $\nabla u_{j}$ is $\arccos \left(\frac{\left|\partial_{i} H_{j}\right|}{\partial_{i} H_{j}}\right)$.

Proof. We apply the geometric Poincaré inequality given as Theorem 3.1 to provide a proof. Ideas and method applied in this proof are strongly motivated by the ones given for the case of the scalar equation by Berestycki, Caffarelli and Nirenberg in [10], Ghoussoub and Gui in [37], Farina, Sciunzi and Valdinoci in 33 and references therein. In addition, for the case of system of equations we refer interested readers to [35, 36] by Ghoussoub, Sire and the author. Note that from boundedness of $\left|\nabla u_{i}\right|$ and $\Phi^{\prime} \in C\left(\mathbb{R}^{+}\right)$in two dimensions we have

$$
\int_{B_{R}} \Phi^{\prime}\left(\left|\nabla u_{i}\right|^{2}\right)\left|\nabla u_{i}\right|^{2} \leq C R^{2},
$$

for any $R>1$. This can be also proved by multiplying (1.15) by ${ }_{R} \zeta_{i}^{2} u_{i}$ and integrating by parts. Straightforward calculations show that for each $i$ we have

$$
\begin{aligned}
& \int_{B_{R} \backslash B_{\sqrt{R}}} \frac{1}{|x|^{2}} \Phi^{\prime}\left(\left|\nabla u_{i}\right|^{2}\right)\left|\nabla u_{i}\right|^{2} d x \\
= & 2 \int_{B_{R} \backslash B_{\sqrt{R}}} \int_{|x|}^{R} \tau^{-3} \Phi^{\prime}\left(\left|\nabla u_{i}\right|^{2}\right)\left|\nabla u_{i}\right|^{2} d \tau d x+\frac{1}{R^{2}} \int_{B_{R} \backslash B_{\sqrt{R}}} \Phi^{\prime}\left(\left|\nabla u_{i}\right|^{2}\right)\left|\nabla u_{i}\right|^{2} \\
\leq & 2 \int_{\sqrt{R}}^{R} \tau^{-3} \int_{B_{\tau}} \Phi^{\prime}\left(\left|\nabla u_{i}\right|^{2}\right)\left|\nabla u_{i}\right|^{2} d x d \tau+\frac{1}{R^{2}} \int_{B_{R}} \Phi^{\prime}\left(\left|\nabla u_{i}\right|^{2}\right)\left|\nabla u_{i}\right|^{2},
\end{aligned}
$$

where we have used the Fubini's theorem. From this and (4.7) we get

$$
\int_{B_{R} \backslash B_{\sqrt{R}}} \frac{\Phi^{\prime}\left(\left|\nabla u_{i}\right|^{2}\right)\left|\nabla u_{i}\right|^{2}}{|x|^{2}} \leq C \log R .
$$

Now for each $i$ set $\eta_{i}$ to be the following standard test function

$$
\eta_{i}(x):= \begin{cases}\frac{1}{2}, & \text { if }|x| \leq \sqrt{R}, \\ \frac{\log R-\log |x|}{\log R}, & \text { if } \sqrt{R}<|x|<R, \\ 0, & \text { if }|x| \geq R .\end{cases}
$$


Note that $\nabla \eta_{i}(x)=-\frac{x}{|x|^{2} \log R}$ on $\sqrt{R}<|x|<R$. Therefore, the right-hand side of the inequality given in theorem 3.1 is of the form

$$
\begin{aligned}
& \sum_{i=1}^{m} \int_{\mathbb{R}^{n}} 2\left|\nabla u_{i}\right|^{2} \Phi^{\prime \prime}\left(\left|\nabla u_{i}\right|^{2}\right)\left|\nabla u_{i} \cdot \nabla \eta_{i}\right|^{2}+\Phi^{\prime}\left(\left|\nabla u_{i}\right|^{2}\right)\left|\nabla u_{i}\right|^{2}\left|\nabla \eta_{i}\right|^{2} \\
= & \sum_{i=1}^{m} \int_{B_{R} \backslash B_{\sqrt{R}}}\left|\nabla u_{i}\right|^{2} \mathcal{A}\left(\nabla u_{i}\right) \nabla \eta_{i} \cdot \nabla \eta_{i} \\
\leq & \frac{C}{\log ^{2} R} \sum_{i=1}^{m} \int_{B_{R} \backslash B_{\sqrt{R}}} \frac{1}{\left|x^{4}\right|}\left|\nabla u_{i}\right|^{2} \mathcal{A}\left(\nabla u_{i}\right) x \cdot x \\
\leq & \frac{C}{\log ^{2} R} \sum_{i=1}^{m} \int_{B_{R} \backslash B_{\sqrt{R}}} \frac{\Phi^{\prime}\left(\left|\nabla u_{i}\right|^{2}\right)\left|\nabla u_{i}\right|^{2}}{|x|^{2}} \\
\leq & \frac{C}{\log R}
\end{aligned}
$$

where we have used (4.8) to conclude the last inequality. From this and the geometric inequality given by (3.6) and the fact that the system is symmetric we get

$$
\begin{aligned}
& \sum_{i \neq j} \int_{B_{\sqrt{R}}}\left(\left|\partial_{j} H_{i}(u)\right|\left|\nabla u_{i}\right|\left|\nabla u_{j}\right|-\partial_{j} H_{i}(u) \nabla u_{i} \cdot \nabla u_{j}\right) \\
& +\sum_{i=1}^{m} \int_{\left\{\left|\nabla u_{i}\right| \neq 0\right\} \cap B_{\sqrt{R}}} \Phi^{\prime}\left(\left|\nabla u_{i}\right|^{2}\right)\left|\nabla u_{i}\right|^{2} \mathcal{K}_{i}^{2} \\
& \quad+\left.\sum_{i=1}^{m} \int_{\left\{\left|\nabla u_{i}\right| \neq 0\right\} \cap B_{\sqrt{R}}}\left[2 \Phi^{\prime \prime}\left(\left|\nabla u_{i}\right|^{2}\right)\left|\nabla u_{i}^{2}\right|+\Phi^{\prime}\left(\left|\nabla u_{i}\right|^{2}\right)\right]\left|\nabla_{T_{i}}\right| \nabla u_{i}\right|^{2} \\
& \leq \\
& \frac{C}{\log R} .
\end{aligned}
$$

Now sending $R \rightarrow \infty$ and the fact that all of the terms in the left-hand side are nonnegative imply that each $u_{i}$ is one dimensional function and $\left|\partial_{j} H_{i}(u)\right|\left|\nabla u_{i}\right|\left|\nabla u_{j}\right|=\partial_{j} H_{i}(u) \nabla u_{i} \cdot \nabla u_{j}$. The latter implies that the angle between $\nabla u_{i}$ and $\nabla u_{j}$ is precisely $\arccos \left(\frac{\left|\partial_{j} H_{i}(u)\right|}{\partial_{j} H_{i}(u)}\right)$ when $i \neq j$. This completes the proof.

Note that in the statement of Theorem 4.1. $\Phi$ does not have to satisfy conditions (A) or (B). However in the next theorem that is in regards to three dimensions one of conditions (A) or (B) is needed. For the case of semilinear systems in two dimensions we refer interested readers to [1] for the construction of two dimensional solutions in the absence of stability and $H$-monotonicity,

Theorem 4.2. Suppose that $u=\left(u_{i}\right)_{i=1}^{m}$ is a classical bounded $H$-monotone solution of symmetric system (1.15) in three dimensions. Let $\Phi$ satisfy one of conditions (A) or (B). Assume also that $\left|\nabla u_{i}\right| \in L^{\infty}\left(\mathbb{R}^{3}\right) \cap$ $W_{\text {loc }}^{1,2}\left(\mathbb{R}^{3}\right)$. Then each $u_{i}$ is a one dimensional function. In addition, the angle between $\nabla u_{i}$ and $\nabla u_{j}$ is $\arccos \left(\frac{\left|\partial_{i} H_{j}\right|}{\partial_{i} H_{j}}\right)$.

Proof. Methods and ideas applied here are strongly motived by the ones given by Ambrosio and Cabré in [6] and Alberti, Ambrosio and Cabré in [2] in the case of a single equation and by Ghoussoub and the author in [35] for the case of systems. We first note that $u$ being $H$-monotone means that $u$ is a stable solution of (1.15). Moreover, the function $v_{i}\left(x_{1}, x_{2}\right):=\lim _{x_{3} \rightarrow \infty} u_{i}\left(x_{1}, x_{2}, x_{3}\right)$ is also a bounded stable solution for (1.15) in $\mathbb{R}^{2}$. Note also that since $u$ is an $H$-monotone solution, the system (1.15) is then orientable. It follows from Theorem 4.1 that each $v_{i}$ is one dimensional and consequently the energy of $v=\left(v_{i}\right)_{i=1}^{m}$ in a two-dimensional ball of radius $R$ is bounded by a multiple of $R$ which implies

$$
\limsup _{t \rightarrow \infty} E\left(u^{t}\right) \leq C R^{2}
$$


where $u^{t}\left(x^{\prime}\right):=u\left(x^{\prime}, x_{n}+t\right)$ for $t \in \mathbb{R}$ and

$$
E_{R}(u)=\int_{B_{R}} \sum_{i=1}^{m} \Phi\left(\left|\nabla u_{i}\right|^{2}\right)-\tilde{H}(u)+c_{u},
$$

for $c_{u}:=\max \tilde{H}(u)$. Applying similar arguments as in the proof of Theorem 2.4 we shall show that

$$
\sum_{i=1}^{m} \int_{B_{R}} \Phi\left(\left|\nabla u_{i}\right|^{2}\right) \leq C R^{2}
$$

where the constant $C$ is independent from $R$. Note that shift function $u^{t}=\left(u_{i}^{t}\right)_{i=1}^{m}$ is also a bounded solution of (1.15) with $\left|\nabla u_{i}^{t}\right| \in L^{\infty}\left(\mathbb{R}^{n}\right)$, i.e.,

$$
-\operatorname{div}\left(\Phi^{\prime}\left(\left|\nabla u_{i}^{t}\right|^{2}\right) \nabla u_{i}^{t}\right)=H_{i}\left(u^{t}\right) \text { in } \mathbb{R}^{n},
$$

and also

$$
\partial_{t} u_{i}^{t}>0>\partial_{t} u_{j}^{t} \quad \text { for all } i \in \bar{I} \text { and } j \in \bar{J} \text { and in } \mathbb{R}^{n} .
$$

Since $u_{i}^{t}$ converges to $v_{i}$ in $C_{l o c}^{1}\left(\mathbb{R}^{n}\right)$ for all $i=1, \cdots, m$, we have

$$
\lim _{t \rightarrow \infty} E\left(u^{t}\right)=E(v) \text {. }
$$

Now, we claim that the following upper bound for the energy holds, for all $t \in \mathbb{R}^{+}$

$$
E_{R}(u) \leq E_{R}\left(u^{t}\right)+M \int_{\partial B_{R}}\left(\sum_{i \in \bar{I}}\left(u_{i}^{t}-u_{i}\right)+\sum_{j \in \bar{J}}\left(u_{j}-u_{j}^{t}\right)\right) d S,
$$

where $M:=\max _{i=1}^{m}\left\{\left.|| \Phi^{\prime}\left(\left|\nabla u_{i}\right|^{2}\right)\left|\nabla u_{i}\right|\right|_{L^{\infty}\left(\mathbb{R}^{n}\right)}\right\}$. Indeed, by differentiating the energy functional along the path $u^{t}$, one gets

$$
\partial_{t} E_{R}\left(u^{t}\right)=\sum_{i=1}^{m} \int_{B_{R}} 2 \Phi^{\prime}\left(\left|\nabla u_{i}^{t}\right|^{2}\right) \nabla u_{i}^{t} \cdot \nabla\left(\partial_{t} u_{i}^{t}\right)-2 H_{i}\left(u^{t}\right) \partial_{t} u_{i}^{t},
$$

Now, multiply (4.12) with $\partial_{t} u^{t}$ and integrate by parts to get

$$
\begin{aligned}
& \int_{B_{R}} \Phi^{\prime}\left(\left|\nabla u_{i}^{t}\right|^{2}\right) \nabla u_{i}^{t} \cdot \nabla\left(\partial_{t} u_{i}^{t}\right) \\
& +\int_{\partial B_{R}} \Phi^{\prime}\left(\left|\nabla u_{i}^{t}\right|^{2}\right) \partial_{\nu} u_{i}^{t} \partial_{t} u_{i}^{t}=\int_{B_{R}} H_{i}\left(u^{t}\right) \partial_{t} u_{i}^{t} .
\end{aligned}
$$

for each $i=1, \cdots, m$. From (4.16) and (4.15) we obtain

$$
\partial_{t} E_{R}\left(u^{t}\right)=2 \sum_{i} \int_{\partial B_{R}} \Phi^{\prime}\left(\left|\nabla u_{i}^{t}\right|^{2}\right) \partial_{\nu} u_{i}^{t} \partial_{t} u_{i}^{t} .
$$

Note that $-M \leq \Phi^{\prime}\left(\left|\nabla u_{i}^{t}\right|^{2}\right) \partial_{\nu} u_{i}^{t} \leq M$ for each $i$ and $\partial_{t} u_{i}^{t}>0>\partial_{t} u_{j}^{t}$ for $i \in \bar{I}$ and $j \in \bar{J}$. Therefore,

$$
\partial_{t} E_{R}\left(u^{t}\right) \geq M \int_{\partial B_{R}}\left(\sum_{j \in \bar{J}} \partial_{t} u_{j}^{t}-\sum_{i \in \bar{I}} \partial_{t} u_{i}^{t}\right) d S .
$$

On the other hand,

$$
\begin{aligned}
E_{R}(u) & =E_{R}\left(u^{t}\right)-\int_{0}^{t} \partial_{t} E_{R}\left(u^{s}\right) d s \\
& \leq E_{R}\left(u^{t}\right)+M \int_{0}^{t} \int_{\partial B_{R}}\left(\sum_{i \in \bar{I}} \partial_{s} u_{i}^{s}-\sum_{j \in \bar{J}} \partial_{s} u_{j}^{s}\right) d S d s \\
& =E_{R}\left(u^{t}\right)+M \int_{\partial B_{R}}\left(\sum_{i \in \bar{I}}\left(u_{i}^{t}-u_{i}\right)+\sum_{j \in \bar{J}}\left(u_{j}-u_{j}^{t}\right)\right) d S .
\end{aligned}
$$


To finish the proof of the theorem just note that $u_{i}<u_{i}^{t}$ and $u_{j}^{t}<u_{j}$ for all $i \in \bar{I}, j \in \bar{J}$ and $t \in \mathbb{R}^{+}$. Moreover, from (4.10) we have $\lim _{t \rightarrow \infty} E_{R}\left(u^{t}\right) \leq C R^{2}$. Therefore, (4.19) yields $E_{R}(u) \leq C\left|\partial B_{R}\right| \leq C R^{2}$. This proves (4.11).

Now, set $\phi_{i}:=\partial_{x_{n}} u_{i}$ and $\psi_{i}:=\nabla u_{i} \cdot \eta$ where $\eta=\left(\eta^{\prime}, 0\right) \in \mathbb{R}^{n-1} \times\{0\}$ and define $\sigma_{i}:=\frac{\psi_{i}}{\phi_{i}}$. Lemma 4.1 implies that $\sigma$ satisfies (4.1). Note that $\phi_{i}^{2} \sigma_{i}^{2}=\psi_{i}^{2} \leq\left|\nabla u_{i}\right|^{2}$. From this and the fact that one of conditions (A) or (B) holds there exists a constant $M$ that is independent from $R$ such that

$$
\begin{aligned}
\sum_{i=1}^{m} \int_{B_{2 R} \backslash B_{R}} \phi_{i}^{2} \sigma_{i}^{2} \mathcal{A}\left(\nabla u_{i}\right)_{R} \Gamma_{i} \cdot{ }_{R} \Gamma_{i} & \leq \sum_{i=1}^{m} \int_{B_{2 R}}\left|\nabla u_{i}\right|^{2} \mathcal{A}\left(\nabla u_{i}\right)_{R} \Gamma_{i} \cdot{ }_{R} \Gamma_{i} \\
& \leq M \sum_{i=1}^{m} \int_{B_{2 R}} \Phi\left(\left|\nabla u_{i}\right|^{2}\right),
\end{aligned}
$$

here we have used the fact that $\left\|_{R} \Gamma_{i}(x)\right\|_{L^{\infty}\left(\mathbb{R}^{n}\right)},\left\|\nabla u_{i}\right\|_{L^{\infty}\left(\mathbb{R}^{n}\right)} \leq C$ for some $C$ that is independent from $R$. We now apply Proposition 4.1 for $h_{i j}=\partial_{j} H_{i}(u) \phi_{i} \phi_{j}$ and the identity function $f$ to conclude that each $\sigma_{i}$ is constant. This implies that each $u_{i}$ is a one-dimensional function. Since $u=\left(u_{i}\right)_{i=1}^{m}$ is a one-dimensional stable solution of (1.15), Theorem 4.1 implies that the angle between $\nabla u_{i}$ and $\nabla u_{j}$ is $\arccos \left(\frac{\left|\partial_{i} H_{j}\right|}{\partial_{i} H_{j}}\right)$.

\section{LIOUVILLE THEOREMS FOR SYMMETRIC SYSTEMS}

For bounded stable solutions of (1.15) up to four dimensions we have the following Liouville theorem as long as each $H_{i}(u)$ is nonnegative.

Theorem 5.1. Suppose that $u=\left(u_{i}\right)_{i=1}^{m}$ is a classical bounded stable solution of symmetric system (1.15) where $H_{i} \geq 0$ for each $i$. Let $\Phi$ satisfy one of conditions $(A)$ or (B). Assume also that $\left|\nabla u_{i}\right| \in L^{\infty}\left(\mathbb{R}^{n}\right) \cap$ $W_{\text {loc }}^{1,2}\left(\mathbb{R}^{n}\right)$. Then each $u_{i}$ must be constant provided $n \leq 4$.

Proof. Multiply both sides of (1.15) with ${ }_{R} \zeta_{i}^{2}\left[u_{i}-\left\|u_{i}\right\|_{L^{\infty}\left(\mathbb{R}^{n}\right)}\right]$ and use assumptions to get

$$
-{ }_{R} \zeta_{i}^{2}\left[u_{i}-\left\|u_{i}\right\|_{L^{\infty}\left(\mathbb{R}^{n}\right)}\right] \operatorname{div}\left(\Phi^{\prime}\left(\left|\nabla u_{i}\right|^{2}\right) \nabla u_{i}\right) \leq 0 \quad \text { in } \mathbb{R}^{n} .
$$

Applying integration by parts, for each $i=1, \cdots, m$ we obtain

$$
\int_{B_{R}} \Phi^{\prime}\left(\left|\nabla u_{i}\right|^{2}\right)\left|\nabla u_{i}\right|^{2} \zeta_{i}^{2} \leq 2 \int_{B_{R}} \Phi^{\prime}\left(\left|\nabla u_{i}\right|^{2}\right)\left|\nabla u_{i}\right|\left|\nabla_{R} \zeta_{i}\right|\left[|| u_{i} \|_{L^{\infty}\left(\mathbb{R}^{n}\right)}-u_{i}\right]_{R} \zeta_{i}
$$

From the Cauchy-Schwarz inequality, for any $R>1$, we get

$$
\sum_{i=1}^{m} \int_{B_{R}} \Phi^{\prime}\left(\left|\nabla u_{i}\right|^{2}\right)\left|\nabla u_{i}\right|^{2} \leq C R^{n-2}
$$

Since $u$ is a stable solution of (1.15) there exists a sequence of functions $\phi=\left(\phi_{i}\right)_{i=1}^{m}$ that each $\phi_{i}$ does not change sign. Similar to the proof of Theorem [5.1] set $\psi_{i}:=\nabla u_{i} \cdot \eta$ where $\eta=\left(\eta^{\prime}, 0\right) \in \mathbb{R}^{n-1} \times\{0\}$ and define $\sigma_{i}:=\frac{\psi_{i}}{\phi_{i}}$. Lemma 4.1 implies that $\sigma=\left(\sigma_{i}\right)_{i=1}^{m}$ satisfies (4.1). Note that $\phi_{i}^{2} \sigma_{i}^{2}=\psi_{i}^{2} \leq\left|\nabla u_{i}\right|^{2}$. From this and the fact that one of conditions (A) or (B) holds there exists a constant $M$ that is independent from $R$ such that

$$
\begin{aligned}
\sum_{i=1}^{m} \int_{B_{R}} \phi_{i}^{2} \sigma_{i}^{2} \mathcal{A}\left(\nabla u_{i}\right)_{R} \Gamma_{i} \cdot{ }_{R} \Gamma_{i} & \leq \sum_{i=1}^{m} \int_{B_{2 R}}\left|\nabla u_{i}\right|^{2} \mathcal{A}\left(\nabla u_{i}\right)_{R} \Gamma_{i} \cdot{ }_{R} \Gamma_{i} \\
& \leq M \sum_{i=1}^{m} \int_{B_{2 R}} \Phi\left(\left|\nabla u_{i}\right|^{2}\right),
\end{aligned}
$$

here we have used the fact that $\left\|_{R} \Gamma_{i}(x)\right\|_{L^{\infty}\left(\mathbb{R}^{n}\right)},\left\|\nabla u_{i}\right\|_{L^{\infty}\left(\mathbb{R}^{n}\right)} \leq C$ for some $C$ that is independent from $R$. Note that due to the general assumption $2 s \Phi^{\prime \prime}(s)+\Phi(s)>0$ when $s>0$ and $\Phi(0)=0$ we have $0 \leq \Phi(s) \leq 2 \Phi^{\prime}(s) s$ for any $s>0$. This implies that

$$
0 \leq \int_{B_{R}} \Phi\left(\left|\nabla u_{i}\right|^{2}\right) \leq 2 \int_{B_{R}} \Phi^{\prime}\left(\left|\nabla u_{i}\right|^{2}\right)\left|\nabla u_{i}\right|^{2} \text { in } \mathbb{R}^{n} .
$$


From (5.5), (5.4) and (5.3) we get

$$
\sum_{i=1}^{m} \int_{B_{R}} \phi_{i}^{2} \sigma_{i}^{2} \mathcal{A}\left(\nabla u_{i}\right)_{R} \Gamma_{i} \cdot{ }_{R} \Gamma_{i} \leq C R^{n-2} .
$$

We now apply Proposition 4.1 for $h_{i j}=\partial_{j} H_{i}(u) \phi_{i} \phi_{j}$ and the identity function $f$ to conclude that each $\sigma_{i}$ is constant when $n \leq 4$. This implies that each $u_{i}$ is a one-dimensional solution of (1.15). Finally (5.6) implies that each $u_{i}$ must be constant.

In the absence of stability condition, there are various Liouvile theorems for solutions of (1.15), at least for the case of $m=1$, in [45, 48] and references therein. For the rest of this section, we mainly focus on the $p$-Laplacian operator that is when $\Phi(s)=\frac{2}{p} s^{\frac{p}{2}}$ and radial solutions of (1.15). For this operator, we provide an optimal Liouville theorem for radial stable solutions. The critical dimension is $n=\frac{4 p}{p-1}+p$ that is much higher than $n=4$ given in Theorem 5.1 for not necessarily radial solutions. This implies that Theorem 5.1 does not seem to be optimal.

Applying the definition of the $p$-Laplacian operator for radial functions in dimension $n$, (1.15) reads

$$
-\left|u_{i}^{\prime}\right|^{p-2}\left((p-1) u_{i}^{\prime \prime}+\frac{n-1}{r} u_{i}^{\prime}\right)=H_{i}(u) \text { for } r \in \mathbb{R}^{+} .
$$

Suppose that $u=\left(u_{i}\right)_{i=1}^{m}$ is a radial stable solution of (5.7) then in the light of (1.27) and (1.26) we have

$$
\begin{aligned}
\int_{\mathbb{R}^{n}}\left|\nabla u_{i}\right|^{p-2}\left(\nabla \phi_{i}, \nabla \zeta_{i}\right) & +(p-2) \int_{\mathbb{R}^{n}}\left|\nabla u_{i}\right|^{p-4}\left(\nabla u_{i}, \nabla \phi_{i}\right)\left(\nabla u_{i}, \nabla \zeta_{i}\right) \\
& =\sum_{j=1}^{m} \int_{\mathbb{R}^{n}} \partial_{j} H_{i}(u) \phi_{j} \zeta_{i} .
\end{aligned}
$$

We now provide the stability inequality for solutions of (1.15) with the $p$-Laplacian operator. This is a particular case of Lemma 3.1 .

Lemma 5.1. Let $u$ denote a stable solution of (1.15). Then

$$
\sum_{i, j=1}^{m} \int \sqrt{\partial_{j} H_{i}(u) \partial_{i} H_{j}(u)} \zeta_{i} \zeta_{j} \leq(p-1) \sum_{i=1}^{m} \int\left|\nabla u_{i}\right|^{p-2}\left|\nabla \zeta_{i}\right|^{2},
$$

for any $\zeta=\left(\zeta_{i}\right)_{i}^{m}$ where $\zeta_{i} \in L^{\infty}\left(\mathbb{R}^{n}\right) \cap W^{1,2}\left(\mathbb{R}^{n}\right)$ with compact support and $1 \leq i \leq m$.

For radial solutions stability inequality is of the following form.

Lemma 5.2. Suppose that $u$ is a radial stable solution of (1.15). Then

$$
\begin{aligned}
& (n-1) \sum_{i=1}^{m} \int_{\mathbb{R}^{n}} \frac{u_{i}^{\prime p}(|x|)}{|x|^{2}} \phi^{2}(x) d x \leq(p-1) \sum_{i=1}^{m} \int_{\mathbb{R}^{n}} u_{i}^{\prime p}(|x|)|\nabla \phi(x)|^{2} d x \\
& +\sum_{i, j=1}^{m} \int_{\mathbb{R}^{n}}\left(\partial_{j} H_{i}(u)-\sqrt{\partial_{j} H_{i}(u) \partial_{i} H_{j}(u)}\right) u_{i}^{\prime}(|x|) u_{j}^{\prime}(|x|) \phi^{2}(x) d x,
\end{aligned}
$$

for all $\phi \in L^{\infty}\left(\mathbb{R}^{n}\right) \cap W^{1,2}\left(\mathbb{R}^{n}\right)$ with compact support.

Proof. Suppose that $u=\left(u_{i}\right)_{i=1}^{m}$ is a radial solutions of (1.15) that is

$$
-\frac{n-1}{r}\left|u_{i}^{\prime}\right|^{p-2} u_{i}^{\prime}-(p-1)\left|u_{i}^{\prime}\right|^{p-2} u_{i}^{\prime \prime}=H_{i}(u),
$$

for $0<r<1$ and $i=1, \cdots, m$. Multiplying the $i^{t h}$ equation of (5.11) with $\left(u_{i}^{\prime} \phi^{2} r^{n-1}\right)^{\prime}$ for $\phi \in L^{\infty}\left(\mathbb{R}^{n}\right) \cap$ $W^{1,2}\left(\mathbb{R}^{n}\right)$ with compact support and performing integration by parts we obtain

$$
\begin{aligned}
\int_{\mathbb{R}^{+}} \sum_{j=1}^{m} \partial_{j} H_{i}(u) u_{j}^{\prime} u_{i}^{\prime} \phi^{2} r^{n-1}= & -\int_{\mathbb{R}^{+}}\left(\frac{n-1}{r}\left|u_{i}^{\prime}\right|^{p-2} u_{i}^{\prime}\right)^{\prime}\left(u_{i}^{\prime} \phi^{2} r^{n-1}\right) \\
& +(p-1) \int_{\mathbb{R}^{+}}\left|u_{i}^{\prime}\right|^{p-2} u_{i}^{\prime \prime}\left(u_{i}^{\prime} \phi^{2} r^{n-1}\right)^{\prime},
\end{aligned}
$$


for all $0<r<1$ and $i=1, \cdots, m$. In addition, straightforward calculations show that

$$
\begin{aligned}
\left(u_{i}^{\prime} \phi^{2} r^{n-1}\right)^{\prime} & =\left(u_{i}^{\prime} \phi^{2}\right)^{\prime} r^{n-1}+(n-1) r^{n-2} u_{i}^{\prime} \phi^{2}, \\
\left(\frac{n-1}{r}\left|u_{i}^{\prime}\right|^{p-2} u_{i}^{\prime}\right)^{\prime} & =-\frac{n-1}{r^{2}}\left|u_{i}^{\prime}\right|^{p-2} u_{i}^{\prime}+(p-1) \frac{n-1}{r}\left|u_{i}^{\prime}\right|^{p-2} u_{i}^{\prime \prime} .
\end{aligned}
$$

Substituting (5.13) and (5.14) in (5.12) we get

$$
\begin{aligned}
\int_{\mathbb{R}^{+}} \sum_{j=1}^{m} \partial_{j} H_{i}(u) u^{\prime}{ }_{j} u_{i}^{\prime} \phi^{2} r^{n-1}= & \int_{\mathbb{R}^{+}} \frac{n-1}{r^{2}}\left|u_{i}^{\prime}\right|^{p} \phi^{2} r^{n-1} \\
& +(p-1) \int_{\mathbb{R}^{+}}\left|u_{i}^{\prime}\right|^{p-2} u_{i}^{\prime \prime}\left(u_{i}^{\prime} \phi^{2}\right)^{\prime} r^{n-1} .
\end{aligned}
$$

Taking sum on the index $i$, we get

$$
\begin{aligned}
\int_{\mathbb{R}^{n}} \sum_{i, j=1}^{m} \partial_{j} H_{i}(u) u^{\prime}{ }_{j} u_{i}^{\prime} \phi^{2}= & (n-1) \sum_{i=1}^{m} \int_{\mathbb{R}^{n}} \frac{\left|u_{i}^{\prime}\right|^{p}}{|x|^{2}} \phi^{2} \\
& +(p-1) \sum_{i=1}^{m} \int_{\mathbb{R}^{n}}\left|u_{i}^{\prime}\right|^{p-2} \nabla u_{i}^{\prime} \cdot \nabla\left(u_{i}^{\prime} \phi^{2}\right) .
\end{aligned}
$$

We now apply the stability inequality (5.8) where $\phi_{i}$ is replaced by $u_{i}^{\prime} \phi$ for a test function $\phi$. Therefore,

$$
\sum_{i, j=1}^{m} \int_{\mathbb{R}^{n}} \sqrt{\partial_{j} H_{i}(u) \partial_{i} H_{j}(u)} u_{i}^{\prime} u_{j}^{\prime} \phi^{2} \leq(p-1) \sum_{i=1}^{m} \int_{\mathbb{R}^{n}}\left|u_{i}^{\prime}\right|^{p-2}\left|\nabla\left(u_{i}^{\prime} \phi\right)\right|^{2} .
$$

Expanding the integrand of the right-hand side we get

$$
\begin{aligned}
(p-1)\left|u_{i}^{\prime}\right|^{p-2}\left(\left|\nabla\left(u_{i}^{\prime} \phi\right)\right|^{2}\right. & =(p-1)\left|u_{i}^{\prime}\right|^{p-2}\left(\left|u_{i}^{\prime}\right|^{2}|\nabla \phi|^{2}+\left|\nabla u_{i}^{\prime}\right|^{2} \phi^{2}+\frac{1}{2} \nabla \phi^{2} \cdot \nabla{u_{i}^{\prime}}^{2}\right) \\
& =(p-1)\left|u_{i}^{\prime}\right|^{p-2}\left(\left|u_{i}^{\prime}\right|^{2}|\nabla \phi|^{2}+\nabla\left(\phi^{2} u_{i}^{\prime}\right) \cdot \nabla u_{i}^{\prime}\right) .
\end{aligned}
$$

From this, (5.12) and (5.17) we get the desired result.

Now, we are ready to classify radial stable solutions of (5.7).

Theorem 5.2. Suppose that $p, m \geq 1$ and $u$ is a radial stable solution of symmetric system (1.15) where $H_{i} \in C^{1}\left(\mathbb{R}^{m}\right)$ whenever $\partial_{j} H_{i}(u)>0$ for all $i, j=1, \cdots, m$. Then, there exist positive constants $r_{0}$ and $C_{n, m, p}$ such that

$$
\sum_{i=1}^{m}\left|u_{i}(r)\right| \geq C_{n, m, p} \begin{cases}r^{\frac{1}{p}\left(p+2-n+2 \sqrt{\frac{n-1}{p-1}}\right),}, & \text { if } n \neq \frac{4 p}{p-1}+p, \\ \log r, & \text { if } n=\frac{4 p}{p-1}+p,\end{cases}
$$

where $r \geq r_{0}$ and $C_{n, m, p}$ is independent from $r$. In addition, assuming that each $u_{i}$ is bounded for $1 \leq i \leq m$, then $n>\frac{4 p}{p-1}+p$ and there is a constant $C_{n, m, p}$ such that

$$
\sum_{i=1}^{m}\left|u_{i}(r)-u_{i}^{\infty}\right| \geq C_{n, m, p} r^{\frac{1}{p}\left(p+2-n+2 \sqrt{\frac{n-1}{p-1}}\right)},
$$

where $r \geq 1$ and $u_{i}^{\infty}:=\lim _{r \rightarrow \infty} u_{i}(r)$ for each $i$.

Proof. Let $u=\left(u_{i}\right)_{i=1}^{m}$ be a radial stable solution of symmetric system (5.7). From Lemma [5.2, the stability inequality becomes

$$
(n-1) \sum_{i=1}^{m} \int_{\mathbb{R}^{n}} \frac{u_{i}^{\prime p}(|x|)}{|x|^{2}} \phi^{2}(x) d x \leq(p-1) \sum_{i=1}^{m} \int_{\mathbb{R}^{n}} u_{i}^{\prime p}(|x|)|\nabla \phi(x)|^{2} d x,
$$

for all $\phi \in L^{\infty}\left(\mathbb{R}^{n}\right) \cap W^{1,2}\left(\mathbb{R}^{n}\right)$ with compact support. Note that the nonlinearity $H=\left(H_{i}\right)_{i=1}^{m}$ does not appear in (5.20). The methods and idea that we apply in this proof are strongly motivated by the ones used 
in 14 16, 19, 54] for the case of a scalar equation, that is when $m=1$, and in [20, 34] for the case of system of equations that is when $m \geq 2$. Test this inequality on the following test function $\phi \in W^{1,2}\left(\mathbb{R}^{+}\right) \cap L^{\infty}\left(\mathbb{R}^{+}\right)$

$$
\phi(t):= \begin{cases}1, & \text { if } 0 \leq t \leq 1 ; \\ t^{-\sqrt{\frac{n-1}{p-1}}}, & \text { if } 1 \leq t \leq r ; \\ \frac{r^{-} \sqrt{\frac{n-1}{p-1}}}{\int_{r}^{R} \frac{d z}{z^{n-1} \sum_{i=1}^{m}\left|u_{i}^{\prime}\right|^{p}(z)}} \int_{t}^{R} \frac{d z}{z^{n-1} \sum_{i=1}^{m}\left|u_{i}^{\prime}\right|^{p}(z)}, & \text { if } r \leq t \leq R ; \\ 0, & \text { if } R \leq t,\end{cases}
$$

for any $1 \leq r \leq R$. Straightforward calculations show that for the given test function $\phi$, the left-hand side of the stability inequality (5.20) has the following lower bound,

$$
(n-1) \int_{0}^{1} \sum_{i=1}^{m}\left|u_{i}^{\prime}\right|^{p}(t) t^{n-3} d t+(n-1) \int_{1}^{r} \sum_{i=1}^{m}\left|u_{i}^{\prime}\right|^{p}(t) t^{-2 \sqrt{\frac{n-1}{p-1}}+n-3} d t .
$$

Similarly we can simplify the right-hand side of the stability inequality using the fact that

$$
\phi^{\prime}(t)= \begin{cases}0, & \text { if } 0 \leq t<1 ; \\ -\sqrt{\frac{n-1}{p-1}} t^{-\sqrt{\frac{n-1}{p-1}}-1}, & \text { if } 1<t<r ; \\ -\frac{r^{-} \sqrt{\frac{n-1}{p-1}}}{\int_{r}^{R} \frac{1}{z^{n-1} \sum_{i=1}^{m}\left|u_{i}^{\prime}\right|^{p}(z)}} \frac{1}{t^{n-1} \sum_{i=1}^{m}\left|u_{i}^{\prime}\right|^{p}(t)}, & \text { if } r \leq t \leq R ; \\ 0, & \text { if } R \leq t .\end{cases}
$$

Substituting this in (5.20), the right-hand side of the inequality would be equivalent to

$$
(n-1) \int_{1}^{r} t^{-2 \sqrt{\frac{n-1}{p-1}}+n-3} \sum_{i=1}^{m}\left|u_{i}^{\prime}\right|^{p}(t) d t+\frac{r^{-2 \sqrt{\frac{n-1}{p-1}}}}{\int_{r}^{R} \frac{d z}{z^{n-1} \sum_{i=1}^{m}\left|u_{i}^{\prime}\right|^{p}(z)}} .
$$

Collecting (5.21) and (5.22), in the light of (5.20), we get

$$
\int_{r}^{R} \frac{d s}{s^{n-1} \sum_{i=1}^{m}\left|u_{i}^{\prime}\right|^{p}(s)} \leq C_{n, m, p} r^{-2 \sqrt{\frac{n-1}{p-1}}} \quad \forall 1 \leq r \leq R,
$$

where the constant $C_{n, m, p}$ is independent from $r, R$ and it is given as

$$
C_{n, m, p}:=\frac{p-1}{(n-1) \int_{0}^{1} \sum_{i=1}^{m}\left|u_{i}^{\prime}\right|^{p}(t) t^{n-3} d t} .
$$

Applying the Hölder's inequality we obtain

$$
\begin{aligned}
\int_{r}^{R} \frac{d s}{s^{\frac{n-1}{p+1}}} & =\int_{r}^{R} \frac{\left(\sum_{i=1}^{m}\left|u_{i}^{\prime}\right|^{p}(s)\right)^{\frac{1}{p+1}}}{s^{\frac{n-1}{p+1}}\left(\sum_{i=1}^{m}\left|u_{i}^{\prime}\right|^{p}(s)\right)^{\frac{1}{p+1}}} d s \\
& \leq\left(\int_{r}^{R} \frac{d s}{s^{n-1} \sum_{i=1}^{m}\left|u_{i}^{\prime}\right|^{p}(s)}\right)^{\frac{1}{p+1}}\left(\int_{r}^{R}\left(\sum_{i=1}^{m}\left|u_{i}^{\prime}\right|^{p}(s)\right)^{\frac{1}{p}} d s\right)^{\frac{p}{p+1}} .
\end{aligned}
$$

From (5.23) we get

$$
\int_{r}^{R} \frac{d s}{s^{\frac{n-1}{p+1}}} \leq C_{n, m, p}^{\frac{1}{p+1}} r^{-\frac{p}{p+1} \sqrt{\frac{n-1}{p-1}}}\left(\sum_{i=1}^{m} \int_{r}^{R}\left|u_{i}^{\prime}(s)\right| d s\right)^{\frac{p}{p+1}} .
$$

Performing straightforward computation for the integral in the left-hand side of (5.25) and taking $R=2 r$, for any $n \geq 2$, one can get

$$
\sum_{i=1}^{m}\left|u_{i}(2 r)-u_{i}(r)\right| \geq C_{n, m, p} r^{\frac{1}{p}\left(p+2-n+2 \sqrt{\frac{n-1}{p-1}}\right)} .
$$


Note that each $u_{i}$ is bounded. Therefore, (5.26) implies

$$
\begin{aligned}
\sum_{i=1}^{m}\left|u_{i}(r)-u_{i}^{\infty}\right| & =\sum_{i=1}^{m} \sum_{k=1}^{\infty}\left|u_{i}\left(2^{k} r\right)-u_{i}\left(2^{k-1} r\right)\right| \\
& \geq C \sum_{k=1}^{\infty}\left(2^{k-1} r\right)^{\frac{1}{p}\left(p+2-n+2 \sqrt{\frac{n-1}{p-1}}\right.} .
\end{aligned}
$$

This proves the second part of the theorem that is (5.19) and $n>\frac{4 p}{p-1}+p$. To prove the first part of the theorem that is (5.18), without loss of generality, we assume that $2 \leq n \leq \frac{4 p}{p-1}+p$. Define $r=2^{k-1} r_{1}$ where $1 \leq r_{1}<2$. Therefore,

$$
\begin{aligned}
\sum_{i=1}^{m}\left|u_{i}(r)\right| & =\sum_{i=1}^{m}\left|u_{i}(r)-u_{i}\left(r_{1}\right)\right|-\sum_{i=1}^{m}\left|u_{i}\left(r_{1}\right)\right| \\
& =\sum_{i=1}^{m} \sum_{j=1}^{k-1}\left|u_{i}\left(2^{j} r_{1}\right)-u_{i}\left(2^{j-1} r_{1}\right)\right|-\sum_{i=1}^{m}\left|u_{i}\left(r_{1}\right)\right| \\
& \geq C_{n, m} \sum_{i=1}^{m} \sum_{j=1}^{k-1}\left(2^{j-1} r_{1}\right)^{\frac{1}{p}\left(p+2-n+2 \sqrt{\frac{n-1}{p-1}}\right)}-\sum_{i=1}^{m}\left|u_{i}\left(r_{1}\right)\right| .
\end{aligned}
$$

This proves the pointwise bound (5.18) when $1<n<\frac{4 p}{p-1}+p$. Finally, when we have dimension $n=\frac{4 p}{p-1}+p$, from the above inequality, we can prove

$$
\sum_{i=1}^{m}\left|u_{i}(r)\right| \geq C_{n, m}(k-1)-\sum_{i=1}^{m}\left|u_{i}\left(r_{1}\right)\right| .
$$

The fact that $k-1=\frac{\log r-\log r_{1}}{\log 2}$ completes the proof.

\section{REFERENCES}

[1] S. Alama, L. Bronsard, C. Gui, Stationary layered solutions in $\mathbb{R}^{2}$ for an Allen-Cahn systems with multiple-well potentials, Calc. Var. Partial Differential Equations 5 (1997) 359-390.

[2] G. Alberti, L. Ambrosio, and X. Cabré, On a long-standing conjecture of E. De Giorgi: symmetry in $3 D$ for general nonlinearities and a local minimality property, Acta Appl. Math. 65 (2001), 9-33.

[3] N. Alikakos, On the structure of phase transition maps for three or more coexisting phases, Geometric Partial Differential Equations. CRM Series, vol. 15. Pisa: Scuola Normale Superiore, pp. 1-31.

[4] N. Alikakos, Some basic facts on the system $\Delta u-W_{u}(u)=0$, Proc. Amer. Math. Soc, Volume 139 (2011) Pages 153-162.

[5] N. Alikakos, G. Fusco, Asymptotic behavior and rigidity results for symmetric solutions of the elliptic system $\Delta u=W_{u}(u)$, To appear in Ann. Sc. Norm. Super. Pisa Cl. Sci.

[6] L. Ambrosio and X. Cabré, Entire solutions of semilinear elliptic equations in $\mathbb{R}^{3}$ and a conjecture of De Giorgi, J. Amer. Math. Soc. 13 (2000), 725-739.

[7] V. I. Arnold, Mathematical Methods of Classical Mechanics, volume 60 of Graduate Texts in Mathematics. Springer-Verlag, New York (1989).

[8] M. T. Barlow, On the Liouville property for divergence form operators, Canadian J. Math. 50 (1998), 487-496.

[9] M. T. Barlow, R. F. Bass and C. Gui, The Liouville property and a conjecture of De Giorgi, Comm. Pure Appl. Math., 53 (2000), no. 8, 1007-1038.

[10] H. Berestycki, L. Caffarelli, and L. Nirenberg, Further qualitative properties for elliptic equations in unbounded domains, Ann. Scuola Norm. Sup. Pisa Cl. Sci. 25 (1997), 69-94.

[11] H. Berestycki, T. Lin, J. Wei and C. Zhao, On Phase-Separation Model: Asymptotics and Qualitative Properties, Archive for Rational Mechanics and Analysis, 208 (2013) 163-200.

[12] H. Brezis, F. Merle, T. Rivire, Quantization effects for $-\Delta u=u\left(1-|u|^{2}\right)$ in $\mathbb{R}^{2}$, Arch. Ration. Mech. Anal. 126 (1) (1994) 35-58. 
[13] X. Cabré, Regularity of minimizers of semilinear elliptic problems up to dimension 4. Comm. Pure Appl. Math. 63 (2010), no. 10, 1362-1380.

[14] X. Cabré, A. Capella, On the stability of radial solutions of semi-linear elliptic equations in all of $\mathbb{R}^{n}$, C. R. Math. Acad. Sci. Paris 338 (2004) 769-774.

[15] X. Cabré and A. Capella, Regularity of radial minimizers and extremal solutions of semilinear elliptic equations, J. Funct. Anal. 238 (2006), no. 2, 709-733.

[16] X. Cabré and M. Sanchon, Semi-stable and extremal solutions of reaction equations involving the $p$ Laplacian, Comm. Pure Appl. Anal. 6 (2007), 43-67.

[17] L. Caffarelli, N. Garofalo, F. Segala, A gradient bound for entire solutions of quasilinear equations and its consequences, Communications on Pure and Applied Mathematics, Vol. XLVII, (1994) 1457-1473.

[18] L. Caffarelli, F. Lin, Singularly perturbed elliptic systems and multi-valued harmonic functions with free boundaries. J. AMS 21 (2008) 847-862.

[19] D. Castorina, P. Esposito, B. Sciunzi, Low dimensional instability for semilinear and quasilinear problems in $\mathbb{R}^{n}$, Communications on Pure and Applied Analysis 20098 (6) 1779-1793.

[20] C. Cowan, M. Fazly, Regularity of the extremal solutions associated to elliptic systems, J. Differential Equations 257 (2014) 4087-4107.

[21] M.G. Crandall and P.H. Rabinowitz, Some continuation and variation methods for positive solutions of nonlinear elliptic eigenvalue problems, Arch. Rat. Mech. Anal., 58 (1975), pp.207-218.

[22] D. Danielli and N. Garofalo, Properties of entire solutions of non-uniformly elliptic equations arising in geometry and in phase transitions, Calc. Var. 15, (2002) 451-491.

[23] E. De Giorgi, Convergence problems for functional and operators, Proceedings of the International Meeting on Recent Methods in Nonlinear Analysis (Rome, 1978), pp. 131-188, Pitagora, Bologna, 1979.

[24] M. del Pino, M. Kowalczyk, J. Wei, On De Giorgi's conjecture in dimension N $\geq 9$. Ann. of Math. (2) (2011). 174:1485-1569.

[25] S. Dipierro, Geometric inequalities and symmetry results for elliptic systems, Discrete and Continuous Dynamical Systems, 33 (2013) 3473-3496.

[26] S. Dipierro, A. Pinamonti, Symmetry results for stable and monotone solutions to fibered systems of PDEs, Communications in Contemporary Mathematics (2014) 1450035.

[27] L. Dupaigne, A. Farina, Stable solutions of $-\Delta u=f(u)$ in $\mathbb{R}^{n}$, J. Eur. Math. Soc. (JEMS) 12 (2010), no. $4,855-882$.

[28] K. Ecker. Regularity theory for mean curvature flow. Birkhauser Verlag, Basel, 2004.

[29] Ivar Ekeland, Convexity Methods in Hamiltonian Mechanics, Ergebnisse der Mathematik und ihrer Grenzgebiete, Springer-Verlag, Volume 191990.

[30] A. Farina, Some remarks on a conjecture of De Giorgi, Calc. Var. Partial Differential Equations, 8, no. 3 (1999), 233-245.

[31] A. Farina. One-dimensional symmetry for solutions of quasilinear equations in $\mathbb{R}^{2}$. Boll. Unione Mat. Ital. Sez. B Artic. Ric. Mat. (8), 6(3):685-692, (2003).

[32] A. Farina, Two results on entire solutions of Ginzburg-Landau system in higher dimensions, J. Functional Analysis 214 No. 2 (2004), pp. $386 ? 395$.

[33] A. Farina, A., B. Sciunzi, E. Valdinoci, Bernstein and de giorgi type problems: New results via a geometric approach. Ann. Sc. Norm. Super. Pisa Cl. Sci. (2008) 7:741-791.

[34] M. Fazly, Rigidity results for stable solutions of symmetric systems, Proc. Amer. Math. Soc. 143 (2015), 5307-5321.

[35] M. Fazly, N. Ghoussoub, De Giorgi type results for elliptic systems, Calc. Var. Partial Differential Equations 47 (2013) 809-823.

[36] M. Fazly, Y. Sire, Symmetry results for fractional elliptic systems and related problems, Communications in PDEs, 40 (2015) 1070-1095.

[37] N. Ghoussoub, C. Gui, On a conjecture of De Giorgi and some related problems, Math. Ann. 311 (1998), no. $3,481-491$.

[38] N. Ghoussoub, C. Gui, About De Giorgi's conjecture in dimensions 4 and 5, Ann. of Math. (2) 157 (2003) 313-334.

[39] C. Gui, Hamiltonian identities for elliptic partial differential equations. J. Funct. Anal. (254) (2008) 904-933. 
[40] W. R. Hamilton, On the application to dynamics of a general mathematical method previously applied to optics. British Association Report, Edinburgh 1834, 513-518.

[41] J. Mawhin, M. Willem, Critical Point Theory and Hamiltonian Systems, Springer-Verlag, Applied Mathematics Science 741989.

[42] L. Modica, A gradient bound and a Liouville theorem for nonlinear Poisson equations, Comm. Pure Appl. Math. 38 (1985), 679-684.

[43] L. Modica, Monotonicity of the energy for entire solutions of semilinear elliptic equations. In Partial differential equations and the calculus of variations, Essays in honor of Ennio De Giorgi, Vol. 2, Birkhauser, Boston,MA, 1989, pp. 843-850.

[44] L. Modica and S. Mortola, Some entire solutions in the plane of nonlinear Poisson equations, Boll. Un. Mat. Ital. 5, 1980, pp. 614-622.

[45] L. A. Peletier and J. Serrin, Gradient estimates and Liouville theorems for quasilinear elliptic equations, Ann. Scuola Norm. Sup. Pisa, 5 (1978), 65-104.

[46] S. I. Pohozaev, Eigenfunctions of the equation $\Delta u+\lambda f(u)=0$, Soviet. Math. Doklady, 6 (1965) 14081411.

[47] O. Savin, Regularity of flat level sets in phase transitions. Ann. of Math. (2) 169 (2009), no. 1, 41-78.

[48] J. Serrin, Liouville theorems for quasilinear elliptic equations, Proc. Convegno Internazionale sul Metodi Valutativi nella Fisica-Matematica, Rome, Accad. Naz. Lincei, Quaderno 217 (1975), 207-215.

[49] R. Schoen, Lecture notes on general relativity, Stanford University, 2009.

[50] R. Schoen and K. Uhlenbeck, A regularity theory for harmonic maps, J. Diff. Geom. 17 No. 2 (1982) 307-335.

[51] L. Simon, Lectures on geometric measure theory, Proceedings of the Centre for Mathematics and its Applications, Vol. 3, Australian National University, Canberra, 1984.

[52] Y. Sire, E. Valdinoci, Fractional Laplacian phase transitions and boundary reactions: A geometric inequality and a symmetry result, J. Funct. Anal. 256 (2009) 1842-1864.

[53] P. Sternberg, K. Zumbrun, A Poincaré inequality with applications to volume-constrained areaminimizing surfaces. J. Reine Angew. Math. (1998) 503:63-85.

[54] S. Villegas, Asymptotic behavior of stable radial solutions of semilinear elliptic equations in $\mathbb{R}^{n}$, J. Math. Pures Appl. (9) 88 (2007), no. 3, 241-250. 